\title{
A RIGID STAMP INDENTATION INTO A SEMIPLANE WITH A CURVATURE-DEPENDENT SURFACE TENSION ON THE BOUNDARY*
}

\author{
JAY R. WALTON ${ }^{\dagger}$ AND ANNA Y. ZEMLYANOVA ${ }^{\ddagger}$
}

\begin{abstract}
It has been shown that taking into account surface mechanics is extremely important for accurate modeling of many physical phenomena such as those arising in nanoscience, fracture propagation, and contact mechanics. This paper is dedicated to a contact problem of a rigid stamp indentation into an elastic isotropic semiplane with curvature-dependent surface tension acting on the boundary of the semiplane. Cases of both frictionless and adhesive contact of the stamp with the boundary of the semiplane are considered. Using the method of integral transforms, each problem is reduced to a system of singular integro-differential equations, which is further reduced to one or two weakly singular integral equations. It has been shown that the introduction of the curvaturedependent surface tension eliminates the classical singularities of the order $1 / 2$ of the stresses and strains at the end-points of the contact interval. The numerical solution of the problem is obtained by approximation of unknown functions with Taylor polynomials.
\end{abstract}

Key words. surface tension, surface elasticity, contact problems, integral equations, microstructure

AMS subject classifications. 74B20, 74G70, 74R10

DOI. $10.1137 / 15 \mathrm{M} 1044096$

1. Introduction. It is well known that particles on the surface of solid objects experience a different environment than those in the bulk. For macro-objects, it is customary to assume that surface energy is small compared to energy in the bulk and, in most cases, can be neglected in the study of the stressed state of the object. This, however, is not true for surface-dominated phenomena such as micro- and nanoscale processes. In this case the ratio of the surface energy to bulk energy is no longer very small, and the surface energy needs to be taken into account in a stress analysis.

Classical linear elasticity lacks interior length scale and, thus, does not allow us to take into account microstructure and surface effects in the material. To overcome this deficiency, various surface energy theories became prominent recently in studies of nanophenomena, especially problems for nanowires, nanorods, nanoinclusions, and also problems of fracture mechanics. Intuitively, surface energy can be understood in terms of a two-dimensional elastic membrane which has been attached to the surface of a three-dimensional bulk substrate. The first rigorous surface elasticity theory of this type was proposed by Gurtin and Murdoch and is described in their seminal works [4], [5]. Later Steigmann and Ogden [20], [21] pointed out that the Gurtin-Murdoch theory cannot be used for a compressive stress-state, and they generalized the Gurtin-Murdoch theory to incorporate curvature dependence of the surface energy. Independently, a different curvature-dependent surface tension theory was proposed by Slattery and coworkers [12], [19]. In this paper we adopt the

\footnotetext{
* Received by the editors October 15, 2015; accepted for publication (in revised form) January 8 , 2016; published electronically March 17, 2016.

http://www.siam.org/journals/siap/76-2/M104409.html

$\dagger$ Department of Mathematics, Texas A\&M University, College Station, TX 77843 (jwalton@math. tamu.edu).

${ }^{\ddagger}$ Department of Mathematics, Kansas State University, Manhattan, KS 66506 (azem@ksu.edu). This author's work was supported by the Simons Foundation through Collaboration Grant for Mathematicians.
} 
latter theory for modeling the surface behavior. The effect of the nanostructure of the material is modeled by the introduction of surface tension $\tilde{\gamma}$ on the boundary of the semiplane, which is assumed to depend linearly on the curvature of the boundary.

The motivation for using this particular theory to study contact problems derives from its successful application to fracture. Since fracture is a nanoscale process, surface mechanics on newly forming fracture surfaces strongly influences the stress and strain fields near the tip of a crack. In particular, it was shown in [17] in the context of the classical Griffith crack problem under pure mode I loading that if the surface stress tensor is chosen to be Eulerian with surface tension being constant, then the resulting crack-tip stress and strain fields lose the traditional strong, squareroot singularity and exhibit only weak, logarithmic singular behavior. Furthermore, it was shown in [17] that under pure mode I loading, if surface tension is chosen to be a function of (linearized) crack surface mean curvature, then the stress and strain fields are bounded in a neighborhood of the crack-tip. However, it was subsequently shown in [22], [25], and [26] that if mixed-mode loading conditions occur at the cracktip (as is the case for an interfacial fracture [25], a curvilinear crack [26], and a classical Griffith crack with mixed-mode far field loading [22]), having surface tension depend upon mean curvature might be insufficient to guarantee bounded crack-tip stress and strain fields; in particular, certain components of the stress tensor might be bounded in a neighborhood of the crack-tip, while other components exhibit a weak logarithmic singularity. It was then shown in [22] that under these conditions, having surface tension depend upon higher tangential gradients of the surface displacement as well as on linearized mean curvature removes the weak crack-tip stress and strain singularities resulting in bounded stress and strain. Additionally, it was shown in [26] that having surface tension depend upon linearized mean curvature removes the oscillating nature of the interfacial crack-tip stress and strain fields predicted under classical (no surface mechanics) linear elastic fracture mechanics (LEFM) theory.

Micro- and nanoindentation tests are frequently used to study microstructuredependent size effects, which motivates the study of contact problems here. It has been noted [7], [11], [13] that in micro- and nanoindentation experimental studies, the obtained values of mechanical parameters significantly depend on the size of the indenter. It has also been observed, both experimentally and through numerical simulation, that this dependency cannot be explained by using bulk theories alone (such as the strain gradient theory), and surface to volume ratio should be taken into account. Different forms of Gurtin-Murdoch surface elasticity have been applied recently in [6], [23], [27], [28] to the contact problems for a semiplane, a semispace, and an elastic layer. In this paper, curvature-dependent surface tension will be assumed to be acting on the boundary of the semiplane.

Cases of both frictionless and adhesive contact of a rigid stamp with a semiplane are studied in the present paper. The outline is as follows. The boundary conditions with the curvature-dependent surface tension are derived for a frictionless contact case in section 2. The mechanical problems are reduced to systems of singular integrodifferential equations which are further reduced to weakly singular integral equations in section 3. In section 4, the effect of the surface tension on the singularities of the stress field near the end-points of the contact zone with the stamp is discussed. The solution procedure for the adhesive contact case is illustrated in section 5. Finally, the numerical procedure is described and the numerical results are obtained for different mechanical and geometric parameters of the problem in sections 6 and 7 . 


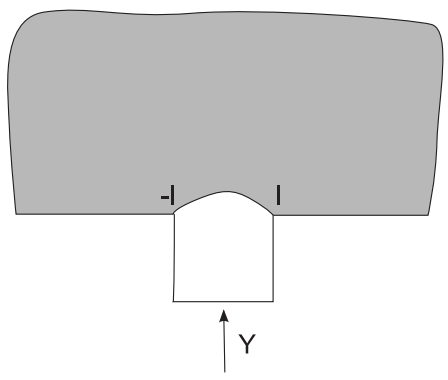

FIG. 1. A rigid stamp indentation into an elastic semiplane.

2. Statement of the problem for a frictionless stamp. Consider a contact problem for a rigid stamp indentation into an isotropic elastic upper semiplane with a Poisson ratio $\nu$ and a shear modulus $\mu$ (Figure 1). Assume that the boundary of the semiplane in an undeformed configuration is aligned with a real axis of the coordinate system and the contact interval along the $x$-axis is $L_{0}=[-l, l]$. The stamp is pressed into the semiplane vertically with a total force $Y$ and a moment about the origin $M=0$ and cannot rotate. It is assumed here that the force $Y$ is large enough so that the actual contact zone of the stamp with the semiplane is the whole interval $L_{0}$. The surface of the semiplane $L_{1}=\mathbb{R} \backslash[-l, l]$ outside of the contact zone is free of stresses. The stresses and the derivatives of the displacements vanish at the infinity of the semiplane. Assume that the surfaces of the semiplane and the stamp are in the frictionless contact and the curvature-dependent surface tension is present on the boundary of the semiplane. It is assumed that the stress field in the bulk is subject to Hooke's law. The surface stress of the form $\mathbf{T}^{(\zeta)}=\tilde{\gamma} \mathbf{P}$ acts on the boundary of the semiplane, where $\mathbf{P}=\mathbf{I}-\mathbf{n} \otimes \mathbf{n}$ is the projection tensor. Then, similarly to [17], the jump momentum condition on the boundary of the semiplane can be taken in the following form:

$$
\operatorname{grad}_{(\zeta)} \tilde{\gamma}+2 \tilde{\gamma} H \mathbf{n}+\llbracket \mathbf{T} \rrbracket \mathbf{n}=\mathbf{0},
$$

where $\tilde{\gamma}$ is surface tension on the boundary of the material, $\mathbf{T}$ denotes the Cauchy stress tensor, $\mathbf{n}$ is the unit normal to the deformed boundary of the semiplane pointing into the bulk of the material, $H=-\frac{1}{2} \operatorname{div}_{(\zeta)} \mathbf{n}$ is the mean curvature, $\operatorname{grad}_{(\zeta)}$ and $\operatorname{div}_{(\zeta)}$ denote the surface gradient and the surface divergence correspondingly, and the double brackets $\llbracket . . . \rrbracket$ denote the jump of the quantity enclosed through the boundary of the semiplane.

Assume that the surface tension acting on the boundary of the semiplane depends linearly on the curvature of the deformed surface [17]:

$$
\tilde{\gamma}=\gamma_{1} \operatorname{div}_{(\zeta)} \mathbf{n}+\gamma_{2}, \quad t \in L_{1},
$$

where $\gamma_{1}, \gamma_{2}$ are real constants. A similar formula with possibly different constants is valid for the material in the contact zone $L_{0}$. The surface mechanics model assumed here treats the material surfaces as dividing surfaces (in the sense of Gibbs) between two distinct material phases; on $L_{0}$, the phases are both solids (the rigid indenter and the elastic semiplane), while on $L_{1}$ the phases are the elastic semiplane and the "vacuum" outside of the material body.

Linearizing (2.1), (2.2), under the assumption that all of the appropriate derivatives of the horizontal $u_{1}$ and vertical $u_{2}$ displacements are small, leads to the following 
modified boundary conditions:

$$
\begin{gathered}
\sigma_{12}(t, 0)=\gamma_{0} u_{2,111}(t, 0), \quad t \in L_{0}, \\
u_{2,1}(t, 0)=g_{2}(t), \quad t \in L_{0},
\end{gathered}
$$

in the contact zone, and

$$
\begin{gathered}
\sigma_{12}(t, 0)=\gamma_{1} u_{2,111}(t, 0), \quad t \in L_{1}, \\
\sigma_{22}(t, 0)=-\gamma_{2} u_{2,11}(t, 0), \quad t \in L_{1},
\end{gathered}
$$

outside of the contact zone, where $\sigma_{22}, \sigma_{12}$ are the normal and the shear stresses acting on the boundary of the semiplane, the subindex 1 after the comma means differentiation by $x$, and $g_{2}(t)$ is the derivative of the function which describes the vertical profile of the rigid stamp. The coefficients $\gamma_{0}$ and $\gamma_{1}$ are allowed to have, in general, different values. No continuity of stresses is assumed through the end-points $t= \pm l$ of the intervals $L_{0}$ and $L_{1}$. Observe that similarly to [17], (2.1), (2.2) are taken in the deformed configuration, while (2.3), (2.4) are in the reference configuration. To obtain the boundary conditions (2.3), (2.4) one needs first to convert (2.1), (2.2) into the equations in the reference (undeformed) configuration and then linearize these equations.

3. Reduction of the problem to one weakly singular integral equation. It is well known that the stresses $\sigma_{22}$ and $\sigma_{12}$, and the derivatives of the displacements $u_{1}, u_{2}$ in the semiplane $S$, can be expressed through two complex functions $\Phi(z), \Psi(z)$ (complex potentials) analytic in $S$ using the following formulas [10]:

$$
\begin{gathered}
\left(\sigma_{22}+i \sigma_{12}\right)(t)=\Phi(t)+\overline{\Phi(t)}+\frac{\overline{d t}}{d t}\left(t \overline{\Phi^{\prime}(t)}+\overline{\Psi(t)}\right), \\
2 \mu \frac{d}{d t}\left(u_{1}+i u_{2}\right)(t)=\kappa \Phi(t)-\overline{\Phi(t)}-\frac{\overline{d t}}{d t}\left(\bar{t} \overline{\Phi^{\prime}(t)}+\overline{\Psi(t)}\right), \quad t \in \mathbb{R} .
\end{gathered}
$$

Here $\kappa=(3-\nu) /(1+\nu)$ for the case of plane stress and $\kappa=3-4 \nu$ for the plane strain. [15]:

Consider the integral representations of the complex potentials proposed by Savruk

$$
\begin{gathered}
\Phi(z)=\frac{1}{2 \pi} \int_{\mathbb{R}} \frac{g^{\prime}(t) d t}{t-z}+\frac{(\kappa+1)^{-1}}{\pi i} \int_{\mathbb{R}} \frac{q(t) d t}{t-z} \\
\Psi(z)=\frac{1}{2 \pi} \int_{\mathbb{R}}\left(\frac{\overline{g^{\prime}(t) d t}}{t-z}-\frac{\bar{t} g^{\prime}(t) d t}{(t-z)^{2}}\right)+\frac{(\kappa+1)^{-1}}{\pi i} \int_{\mathbb{R}}\left(\frac{\kappa \overline{q(t) d t}}{t-z}-\frac{\bar{t} q(t) d t}{(t-z)^{2}}\right), \quad z \in S,
\end{gathered}
$$

where the functions $q(t)$ and $g^{\prime}(t)$, defined on the real axis $\mathbb{R}$, represent the jumps of the stresses and the derivatives of the displacements through the real axis $\mathbb{R}$ :

$$
\begin{gathered}
2 q(t)=\left(\sigma_{22}+i \sigma_{12}\right)^{+}(t)-\left(\sigma_{22}+i \sigma_{12}\right)^{-}(t), \quad t \in \mathbb{R} \\
\frac{i(\kappa+1)}{2 \mu} g^{\prime}(t)=\frac{d}{d t}\left(u_{1}+i u_{2}\right)^{+}(t)-\frac{d}{d t}\left(u_{1}+i u_{2}\right)^{-}(t), \quad t \in \mathbb{R},
\end{gathered}
$$

Copyright (c) by SIAM. Unauthorized reproduction of this article is prohibited. 
where superscripts "+" and "-" correspond to the parameters in the upper and lower semiplanes, respectively.

Since there is no physical material present in the lower semiplane, the complex potentials there can be chosen formally with a certain degree of arbitrariness according to the convenience of the solution to the problem. In this problem, we formally extend the domain of the definition of the complex potentials $\Phi(z), \Psi(z)$ from the upper semiplane $S$ to the full complex plane in such a way that the derivatives of the displacements are equal to zero on the boundary of the lower semiplane:

$$
\frac{d}{d t}\left(u_{1}+i u_{2}\right)^{-}(t)=0, \quad t \in \mathbb{R}
$$

Observe that due to the uniqueness of the solution of the second fundamental problem of elasticity [10], it follows that the only solution to the boundary value problem (3.6) in the lower semiplane is the trivial solution (up to the rigid motions of the semiplane). Hence, the stresses also must be equal to zero on the boundary of the lower semiplane:

$$
\left(\sigma_{22}+i \sigma_{12}\right)^{-}(t)=0, \quad t \in \mathbb{R} .
$$

Substituting the integral representations (3.3) into the Muskhelishvili formulas (3.1), (3.2) and remembering that $t=\bar{t}$ on the real axis allow us to obtain simple formulas for the stresses and the derivatives of the displacements on the real axis:

$$
\begin{gathered}
\left(\sigma_{22}+i \sigma_{12}\right)^{ \pm}(t)= \pm q(t)+\frac{1}{\pi} \int_{\mathbb{R}} \frac{g^{\prime}(\tau) d \tau}{\tau-t}-\frac{\kappa-1}{\pi i(\kappa+1)} \int_{\mathbb{R}} \frac{q(\tau) d \tau}{\tau-t}, \quad t \in \mathbb{R} \\
2 \mu \frac{d}{d t}\left(u_{1}+i u_{2}\right)^{ \pm}(t)= \pm \frac{i(\kappa+1)}{2} g^{\prime}(t)+\frac{\kappa-1}{2 \pi} \int_{\mathbb{R}} \frac{g^{\prime}(\tau) d \tau}{\tau-t}+\frac{2 \kappa}{\pi i(\kappa+1)} \int_{\mathbb{R}} \frac{q(\tau) d \tau}{\tau-t} \\
t \in \mathbb{R}
\end{gathered}
$$

Substitute (3.6), (3.7) into (3.4), (3.5), and then into the boundary conditions $(2.3),(2.4)$, and arrive at the following new boundary conditions:

$$
\begin{gathered}
\operatorname{Im} q_{0}(t)=\frac{\gamma_{0}}{2} g_{2}^{\prime \prime}(t), \quad \operatorname{Re} g_{0}^{\prime}(t)=\frac{2 \mu}{\kappa+1} g_{2}(t), \quad t \in L_{0}, \\
\operatorname{Im} q_{1}(t)=\frac{\gamma_{1}(\kappa+1)}{4 \mu} \operatorname{Re} g_{1}^{\prime \prime \prime}(t), \quad \operatorname{Re} q_{1}(t)=-\frac{\gamma_{2}(\kappa+1)}{4 \mu} \operatorname{Re} g_{1}^{\prime \prime}(t), \quad t \in L_{1} .
\end{gathered}
$$

Here and further, the subindex " 0 " denotes the values of the functions $g^{\prime}(t), q(t)$, and their real and imaginary parts, and their derivatives on the contact interval $L_{0}$, while the subindex " 1 " denotes the values of these functions on the interval $L_{1}$ outside of the contact interval. The functions $g^{\prime}(t), q(t)$ without any subindices are presumed to be defined on the whole real axis $\mathbb{R}$ :

$$
g^{\prime}(t)=\left\{\begin{array}{l}
g_{0}^{\prime}(t), \quad t \in L_{0}, \\
g_{1}^{\prime}(t), \quad t \in L_{1},
\end{array} \quad q(t)= \begin{cases}q_{0}(t), \quad t \in L_{0} \\
q_{1}(t), \quad t \in L_{1}\end{cases}\right.
$$


The condition (3.6) then can be rewritten with the help of formulas (3.9) as

$$
\begin{aligned}
& \frac{\kappa+1}{2} \operatorname{Im} g^{\prime}(t)+\frac{\kappa-1}{2 \pi} \int_{\mathbb{R}} \frac{\operatorname{Re} g^{\prime}(\tau) d \tau}{\tau-t}+\frac{2 \kappa}{\pi(\kappa+1)} \int_{\mathbb{R}} \frac{\operatorname{Im} q(\tau) d \tau}{\tau-t}=0, \quad t \in \mathbb{R} \\
& -\frac{\kappa+1}{2} \operatorname{Re} g^{\prime}(t)+\frac{\kappa-1}{2 \pi} \int_{\mathbb{R}} \frac{\operatorname{Im} g^{\prime}(\tau) d \tau}{\tau-t}-\frac{2 \kappa}{\pi(\kappa+1)} \int_{\mathbb{R}} \frac{\operatorname{Re} q(\tau) d \tau}{\tau-t}=0, \quad t \in \mathbb{R}
\end{aligned}
$$

where the real and the imaginary parts of the equation have been separated. Assuming that the unknown functions in $(3.12),(3.13)$ belong to the space $L^{2}(\mathbb{R})$, take the Fourier transform

$$
\mathcal{F}(f)=\int_{\mathbb{R}} e^{-2 \pi i s t} f(t) d t
$$

of (3.12), (3.13), and use the following relationship between the Fourier and the Hilbert transform [8]:

$$
\mathcal{F}(H(f))=-i \operatorname{sign} s \mathcal{F}(f),
$$

where

$$
H(f)=\frac{1}{\pi} \int_{\mathbb{R}} \frac{f(\tau) d \tau}{\tau-t} .
$$

Then the unknown function $\mathcal{F}\left(\operatorname{Im} g^{\prime}(t)\right)$ can be excluded from the resulting equations, and the system (3.12), (3.13) can be reduced to only one equation:

$$
\begin{aligned}
& -\mathcal{F}\left(\operatorname{Re} g_{1}^{\prime}(t)\right)-i \operatorname{sign} s \frac{\gamma_{2}(\kappa+1)}{4 \mu} \mathcal{F}\left(\operatorname{Re} g_{1}^{\prime \prime}(t)\right)+\frac{\gamma_{1}(\kappa-1)}{4 \mu} \mathcal{F}\left(\operatorname{Re} g_{1}^{\prime \prime \prime}(t)\right) \\
& =\frac{2 \mu}{\kappa+1} \mathcal{F}\left(g_{2}(t)\right)-i \operatorname{sign} s \mathcal{F}\left(\operatorname{Re} q_{0}(t)\right)+\frac{\gamma_{0}(\kappa-1)}{2(\kappa+1)} \mathcal{F}\left(g_{2}^{\prime \prime}(t)\right) .
\end{aligned}
$$

Assuming that $\operatorname{Re} g^{\prime}(t) \rightarrow 0, \operatorname{Re} g^{\prime \prime}(t) \rightarrow 0$ as $t \rightarrow \pm \infty$ (this fact is verified in the appendix), integrate by parts:

$$
\begin{gathered}
\mathcal{F}\left(\operatorname{Re} g_{1}^{\prime \prime}(t)\right)=-e^{-2 \pi i l s} \operatorname{Re} g_{1}^{\prime}(l)+e^{2 \pi i l s} \operatorname{Re} g_{1}^{\prime}(-l)+2 \pi i s \mathcal{F}\left(\operatorname{Re} g_{1}^{\prime}(t)\right), \\
\mathcal{F}\left(\operatorname{Re} g_{1}^{\prime \prime \prime}(t)\right)=-e^{-2 \pi i l s} \operatorname{Re} g_{1}^{\prime \prime}(l)+e^{2 \pi i l s} \operatorname{Re} g_{1}^{\prime \prime}(-l) \\
+2 \pi i s\left(-e^{-2 \pi i l s} \operatorname{Re} g_{1}^{\prime}(l)+e^{2 \pi i l s} \operatorname{Re} g_{1}^{\prime}(-l)\right)+(2 \pi i s)^{2} \mathcal{F}\left(\operatorname{Re} g_{1}^{\prime}(t)\right) .
\end{gathered}
$$

Substituting the second formula (3.15) into (3.14) allows us to solve (3.14) for the Fourier transform $\mathcal{F}\left(\operatorname{Re} g_{1}^{\prime}(t)\right)$ :

$$
\begin{aligned}
& \mathcal{F}\left(\operatorname{Re} g_{1}^{\prime}(t)\right)=\left(s^{2}-\frac{\gamma_{2}(\kappa+1)}{2 \gamma_{1}(\kappa-1) \pi}|s|+\frac{\mu}{\gamma_{1}(\kappa-1) \pi^{2}}\right)^{-1} \\
& \times\left\{-\left(\frac{\gamma_{2}(\kappa+1)}{4 \gamma_{1}(\kappa-1) \pi^{2}} i \operatorname{sign} s+\frac{s}{2 \pi i}\right)\left(-C_{1} e^{-2 \pi i l s}+C_{2} e^{2 \pi i l s}\right)\right. \\
&+\frac{1}{4 \pi^{2}}\left(-C_{3} e^{-2 \pi i l s}+C_{4} e^{2 \pi i l s}\right)-\frac{2 \mu^{2}}{\gamma_{1}\left(\kappa^{2}-1\right) \pi^{2}} \mathcal{F}\left(g_{2}(t)\right) \\
&\left.+\frac{\mu}{\gamma_{1}(\kappa-1) \pi^{2}} i \operatorname{sign} s \mathcal{F}\left(\operatorname{Re} q_{0}(t)\right)-\frac{\mu \gamma_{0}}{2 \gamma_{1}(\kappa+1) \pi^{2}} \mathcal{F}\left(g_{2}^{\prime \prime}(t)\right)\right\},
\end{aligned}
$$

Copyright $@$ by SIAM. Unauthorized reproduction of this article is prohibited. 
where the constants $C_{1}, C_{2}, C_{3}$, and $C_{4}$ are defined by $C_{1}=\operatorname{Re} g_{1}^{\prime}(l), C_{2}=\operatorname{Re} g_{1}^{\prime}(-l)$, $C_{3}=\operatorname{Re} g_{1}^{\prime \prime}(l)$, and $C_{4}=\operatorname{Re} g_{1}^{\prime \prime}(-l)$.

Substituting formula (3.16) into formulas (3.15) allows us to find the Fourier transform $\mathcal{F}\left(\operatorname{Re} g_{1}^{\prime \prime \prime}(t)\right)$ as well:

$$
\begin{gathered}
\mathcal{F}\left(\operatorname{Re} g_{1}^{\prime \prime \prime}(t)\right)=\left(s^{2}-\frac{\gamma_{2}(\kappa+1)}{2 \gamma_{1}(\kappa-1) \pi}|s|+\frac{\mu}{\gamma_{1}(\kappa-1) \pi^{2}}\right)^{-1} \\
\times\left\{\frac{2 \mu i s}{\gamma_{1}(\kappa-1) \pi}\left(-C_{1} e^{-2 \pi i l s}+C_{2} e^{2 \pi i l s}\right)\right. \\
+\left(-\frac{\gamma_{2}(\kappa+1)}{2 \gamma_{1}(\kappa-1) \pi}|s|+\frac{\mu}{\gamma_{1}(\kappa-1) \pi^{2}}\right)\left(-C_{3} e^{-2 \pi i l s}+C_{4} e^{2 \pi i l s}\right) \\
\left.+\frac{8 \mu^{2} s^{2}}{\gamma_{1}\left(\kappa^{2}-1\right)} \mathcal{F}\left(g_{2}(t)\right)-\frac{4 \mu i s^{2} \operatorname{sign} s}{\gamma_{1}(\kappa-1)} \mathcal{F}\left(\operatorname{Re} q_{0}(t)\right)+\frac{2 \mu \gamma_{0} s^{2}}{\gamma_{1}(\kappa+1)} \mathcal{F}\left(g_{2}^{\prime \prime}(t)\right)\right\} .
\end{gathered}
$$

Using the convolution formula for Fourier transforms [8], it is possible to invert the Fourier transforms (3.16), (3.17):

$$
\begin{gathered}
-\frac{1}{2 \pi} C_{2}\left(K_{3}(t+l)-\frac{\gamma_{2}(\kappa+1)}{2 \gamma_{1}(\kappa-1) \pi} K_{2}(t+l)\right)+\frac{1}{4 \pi^{2}}\left(-C_{3} K_{1}(t-l)+C_{4} K_{1}(t+l)\right) \\
-\frac{2 \mu^{2}}{\gamma_{1}\left(\kappa^{2}-1\right) \pi^{2}} \int_{L_{0}} g_{2}(\tau) K_{1}(t-\tau) d \tau-\frac{\mu}{\gamma_{1}(\kappa-1) \pi^{2}} \int_{L_{0}} \operatorname{Re} q_{0}(\tau) K_{2}(t-\tau) d \tau \\
-\frac{\mu \gamma_{0}}{2 \gamma_{1}(\kappa+1) \pi^{2}} \int_{L_{0}} g_{2}^{\prime \prime}(\tau) K_{1}(t-\tau) d \tau, \quad t \in L_{1}, \\
\quad \operatorname{Re} g_{1}^{\prime \prime \prime}(t)=-\frac{2 \mu}{\gamma_{1}(\kappa-1)}\left(-C_{1} K_{3}(t-l)+C_{2} K_{3}(t+l)\right) \\
+C_{3}\left(\frac{\gamma_{2}(\kappa+1)}{2 \gamma_{1}(\kappa-1) \pi} K_{4}(t-l)-\frac{\mu}{\gamma_{1}(\kappa-1) \pi^{2}} K_{1}(t-l)\right) \\
-C_{4}\left(\frac{\gamma_{2}(\kappa+1)}{2 \gamma_{1}(\kappa-1) \pi} K_{4}(t+l)-\frac{\mu}{\gamma_{1}(\kappa-1) \pi^{2}} K_{1}(t+l)\right) \\
+\frac{8 \mu^{2}}{\gamma_{1}\left(\kappa^{2}-1\right)} \int_{L_{0}} g_{2}(\tau)\left(\frac{\gamma_{2}(\kappa+1)}{2 \gamma_{1}(\kappa-1) \pi} K_{4}(t-\tau)-\frac{\mu}{\gamma_{1}(\kappa-1) \pi^{2}} K_{1}(t-\tau)\right) d \tau \\
+\frac{4 \mu}{\gamma_{1}(\kappa-1)} \int_{L_{0}} \operatorname{Re} q_{0}(\tau)\left(\frac{\gamma_{2}(\kappa+1)}{2 \gamma_{1}(\kappa-1) \pi} K_{3}(t-\tau)-\frac{\mu}{\gamma_{1}(\kappa-1) \pi^{2}} K_{2}(t-\tau)\right) d \tau \\
+\frac{2 \mu \gamma_{0}}{\gamma_{1}(\kappa+1)} \int_{L_{0}} g_{2}^{\prime \prime}(\tau)\left(\frac{\gamma_{2}(\kappa+1)}{2 \gamma_{1}(\kappa-1) \pi} K_{4}(t-\tau)-\frac{\mu}{\gamma_{1}(\kappa-1) \pi^{2}} K_{1}(t-\tau)\right) d \tau \\
\frac{4 \mu}{\gamma_{1}(\kappa-1) \pi} \int_{L_{0}} \frac{\operatorname{Re} q_{0}(\tau) d \tau}{\tau-t}, \quad t \in L_{1},
\end{gathered}
$$

Copyright (c) by SIAM. Unauthorized reproduction of this article is prohibited. 
where the kernels $K_{j}(t), j=1,2,3,4$, are defined by

$$
\begin{gathered}
K_{1}(t+c)=\mathcal{F}^{-1}\left(\frac{e^{2 \pi i c s}}{(|s|-a)^{2}+b^{2}}\right)=2 \int_{0}^{\infty} \frac{\cos 2 \pi s(t+c) d s}{(s-a)^{2}+b^{2}}, \\
K_{2}(t+c)=-i \mathcal{F}^{-1}\left(\frac{\operatorname{sign} s e^{2 \pi i c s}}{(|s|-a)^{2}+b^{2}}\right)=2 \int_{0}^{\infty} \frac{\sin 2 \pi s(t+c) d s}{(s-a)^{2}+b^{2}}, \\
K_{3}(t+c)=-i \mathcal{F}^{-1}\left(\frac{s e^{2 \pi i c s}}{(|s|-a)^{2}+b^{2}}\right)=2 \int_{0}^{\infty} \frac{s \sin 2 \pi s(t+c) d s}{(s-a)^{2}+b^{2}}, \\
K_{4}(t+c)=\mathcal{F}^{-1}\left(\frac{|s| e^{2 \pi i c s}}{(|s|-a)^{2}+b^{2}}\right)=2 \int_{0}^{\infty} \frac{s \cos 2 \pi s(t+c) d s}{(s-a)^{2}+b^{2}}, \\
a=\frac{\gamma_{2}(\kappa+1)}{4 \gamma_{1}(\kappa-1) \pi}, \quad a^{2}+b^{2}=\frac{\mu}{\gamma_{1}(\kappa-1) \pi^{2}} .
\end{gathered}
$$

Here it is assumed that $16 \mu>\gamma_{1} \gamma_{2}\left(\kappa^{2}-1\right)$ (and, hence, $b$ is a real number). In practice, the shear modulus $\mu$ for many industrial materials (such as metal alloys) satisfies the condition $\mu \geq 40$, and the parameter $\kappa$ always satisfies the condition $\kappa<3$. Hence, it is sufficient to consider the parameters $\gamma_{1}, \gamma_{2}$ such that $\gamma_{1} \gamma_{2}<80$, which is a physically reasonable condition for surface tension parameters.

It is possible to show that the functions $K_{1}(t)$ and $K_{2}(t)$ are Hölder continuous with any exponent $\lambda, 0<\lambda<1$, on the whole real axis and belong to the space $L^{2}(\mathbb{R})$. The function $K_{3}(t)$ belongs to the space $L^{2}(\mathbb{R})$, is Hölder continuous with any exponent $\lambda, 0<\lambda<1$, on $(-\infty, 0)$ and $(0, \infty)$ separately, and has a discontinuity of the first kind at $t=0$. The function $K_{4}(t)$ belongs to the space $L^{2}(\mathbb{R})$, is Hölder continuous with any exponent $\lambda, 0<\lambda<1$, on $(-\infty, 0)$ and $(0, \infty)$ separately, and has a logarithmic singularity at $t=0$. The proofs of these properties can be found in the appendix.

To regularize the system of the singular integro-differential equations (3.12), (3.13), solve (3.14) for $\mathcal{F}\left(\operatorname{Re} q_{0}(t)\right)$ and invert the Fourier transform:

$$
\begin{aligned}
& \operatorname{Re} q_{0}(t)=\frac{1}{\pi} \int_{L} \frac{\operatorname{Re} g_{1}^{\prime}(\tau) d \tau}{\tau-t}-\frac{\gamma_{2}(\kappa+1)}{4 \pi \mu} \int_{L} \frac{\operatorname{Re} g_{1}^{\prime \prime \prime}(\tau) d \tau}{\tau-t} \\
& +\frac{2 \mu}{\kappa+1} \int_{L_{0}} \frac{g_{2}(\tau) d \tau}{\tau-t}+\frac{\gamma_{0}(\kappa-1)}{2(\kappa+1)} \int_{L_{0}} \frac{g_{2}^{\prime \prime}(\tau) d \tau}{\tau-t}, \quad t \in L_{0} .
\end{aligned}
$$

The final step is to substitute the formulas (3.18), (3.19) into (3.21). This reduces the frictionless contact problem under consideration to one weakly singular equation for the function $\operatorname{Re} q_{0}(t)$ on the finite interval $L_{0}$ :

$$
\operatorname{Re} q_{0}(t)=-\frac{1}{\pi^{2}} \int_{L_{0}} \log \left|\frac{(l-\tau)(l+t)}{(l+\tau)(l-t)}\right| \frac{\operatorname{Re} q_{0}(\tau) d \tau}{\tau-t}-\frac{\gamma_{2}(\kappa+1)}{2 \gamma_{1}(\kappa-1) \pi^{2}}
$$

$$
\begin{aligned}
& \times \int_{L_{0}} \operatorname{Re} q_{0}(\tau) d \tau \int_{L} \frac{K_{2}\left(\tau_{1}-\tau\right) d \tau_{1}}{\tau_{1}-t}-\frac{\mu \gamma_{2}}{\gamma_{1}(\kappa-1) \pi^{2}} \int_{L_{0}} g_{2}(\tau) d \tau \int_{L} \frac{K_{4}\left(\tau_{1}-\tau\right) d \tau_{1}}{\tau_{1}-t} \\
& -\frac{\gamma_{0} \gamma_{2}}{4 \gamma_{1} \pi^{2}} \int_{L_{0}} g_{2}^{\prime \prime}(\tau) d \tau \int_{L} \frac{K_{4}\left(\tau_{1}-\tau\right) d \tau_{1}}{\tau_{1}-t}+\frac{2 \mu}{\pi(\kappa+1)} \int_{L_{0}} \log \left|\frac{(l-\tau)(l+t)}{(l+\tau)(l-t)}\right| \frac{g_{2}(\tau) d \tau}{\tau-t}
\end{aligned}
$$




$$
\begin{gathered}
+\frac{\gamma_{0}(\kappa-1)}{2 \pi(\kappa+1)} \int_{L_{0}} \log \left|\frac{(l-\tau)(l+t)}{(l+\tau)(l-t)}\right| \frac{g_{2}^{\prime \prime}(\tau) d \tau}{\tau-t} \\
+\frac{\gamma_{2}(\kappa+1)}{4 \pi^{3} \gamma_{1}(\kappa-1)}\left(-C_{1} \int_{L} \frac{K_{2}(\tau-l) d \tau}{\tau-t}+C_{2} \int_{L} \frac{K_{2}(\tau+l) d \tau}{\tau-t}\right) \\
+\frac{\gamma_{2}(\kappa+1)}{8 \pi \mu}\left(-C_{3} \int_{L} \frac{K_{4}(\tau-l) d \tau}{\tau-t}+C_{4} \int_{L} \frac{K_{4}(\tau+l) d \tau}{\tau-t}\right), \quad t \in L_{0} .
\end{gathered}
$$

The kernel of this equation belongs to the space $L^{2}([-l, l] \times[-l, l])$, is Hölder continuous on $(-l, l)$ with any exponent $0<\lambda<1$, and has at most singularity of logarithm-squared type at the end-points $t= \pm l[2]$. Assuming that the functions $g_{2}(t), g_{2}^{\prime \prime}(t)$ are Hölder continuous on $L_{0}$ up to the ends, it follows that the right-hand side of (3.22) also belongs to the space $L^{2}(-l, l)$, is Hölder continuous on $(-l, l)$, and is a weakly singular function with a singularity having at most logarithm-squared type at the end-points $t= \pm l$. Then it follows from the theory of Fredholm equations [9] that the solution $\operatorname{Re} q_{0}(t)$ to $(3.22)$ exists, is unique for almost all values of the parameters, and is Hölder continuous on $L_{0}$ with the possible exception of the end-points $t= \pm l$. Observe that then from the formula (3.18), it follows that the function $\operatorname{Re} g^{\prime}(t)$ also belongs to the space $L^{2}(\mathbb{R} \backslash[-l, l])$ and is Hölder continuous. Similar conclusions can be made for the functions $\operatorname{Re} g^{\prime \prime}(t)$ and $\operatorname{Re} g^{\prime \prime \prime}(t)$. Hence, all the operations which led to (3.22) are justified.

The solution $\operatorname{Re} q_{0}(t)$ to $(3.22)$ contains four real constants $C_{j}, j=1,2,3,4$. Four additional conditions for finding these constants will be given later.

4. Singularities of the solution at the end-points of contact zone with the stamp $\boldsymbol{x}= \pm \boldsymbol{l}$. From the previous studies [16], [17], [25], [26], it is known that taking into account the curvature-dependent surface tension in the form (2.2) on the boundary of an interface or a noninterface crack leads to the elimination of the classical power singularities of the order $1 / 2$ at the crack-tips and oscillating singularities of the pure imaginary order in the case of an interface crack. Weaker logarithmic singularities may still be present at the tips of the crack. Let us investigate the singularities of the stresses and strains at the end-points of the contact interval for the case of the frictionless contact of the stamp with the semiplane.

Assume that the function $g_{2}(x)$ and its second derivative $g_{2}^{\prime \prime}(x)$ are Hölder continuous and do not have singularities of any kind at the end-points of the contact zone $x= \pm l$. By excluding the unknown $\operatorname{Im} g^{\prime}(t)$, the system (3.12), (3.13) can be reduced to one singular integral equation:

$$
-\operatorname{Re} g^{\prime}(t)+\frac{\kappa-1}{\kappa+1} \operatorname{Im} q(t)-\frac{1}{\pi} \int_{\mathbb{R}} \frac{\operatorname{Re} q(\tau) d \tau}{\tau-t}=0, \quad t \in \mathbb{R} .
$$

Consider this equation on the interval $L_{0}$. Observe that the unknowns $\operatorname{Re} g^{\prime}(t)=$ $\operatorname{Re} g_{0}^{\prime}(t)$ and $\operatorname{Im} q(t)=\operatorname{Im} q_{0}(t), t \in L_{0}$, can be expressed through the functions $g_{2}(t)$ and $g_{2}^{\prime \prime}(t)$, and hence are bounded at the end-points $t= \pm l$ of the interval $L_{0}$. Also observe that since all the unknown functions can have at most integrable singularities at $t= \pm l$, the function $\operatorname{Im} q_{1}(t)=\frac{\gamma_{1}(\kappa+1)}{4 \mu} \operatorname{Re} g_{1}^{\prime \prime \prime}(t)$ has at most integrable singularities at those points. Then it follows that the functions $\operatorname{Re} g_{1}^{\prime}(t), \operatorname{Re} g_{1}^{\prime \prime}(t)$, and $\operatorname{Re} q_{1}(t)$ are bounded at $t= \pm l$.

Since the functions $\operatorname{Re} g_{0}^{\prime}(t)$ and $\operatorname{Im} q_{0}(t)$ are bounded, it follows from (4.1) that the integral

$$
\int_{\mathbb{R}} \frac{\operatorname{Re} q(\tau) d \tau}{\tau-t}
$$


is bounded at the end-points $t= \pm l$. Then, there is no discontinuity at these points, and hence the function $\operatorname{Re} q_{0}(t)$ is also bounded at $t= \pm l$, and, in addition, it satisfies the conditions

$$
\operatorname{Re} q_{0}( \pm l)=\operatorname{Re} q_{1}( \pm l) .
$$

From (4.1) and the fact that $\operatorname{Re} g_{1}^{\prime}(t)$ is bounded at the end-points $t= \pm l$, it follows that the function $\operatorname{Im} q_{1}(t)$ is also bounded at these points. Finally, from (3.12), obtain that the functions $\operatorname{Im} g_{0}^{\prime}(t)$ and $\operatorname{Im} g_{1}^{\prime}(t)$ have at most logarithmic type of singularities at $t= \pm l$. Hence, the stresses and the derivatives of the displacements have the following behavior at the points $t= \pm l$ :

$$
\begin{gathered}
\sigma_{12}(t, 0)=O(1), \quad \sigma_{22}(t, 0)=O(1), \quad t \rightarrow \pm l, \\
u_{1,1}(t, 0)=O(\log |t \pm l|), \quad t \rightarrow \pm l, \\
u_{2,1}(t, 0)=O(1), \quad t \rightarrow \pm l,
\end{gathered}
$$

where either all the top signs or all the bottom signs need to be taken.

Therefore, it has been shown that similarly to the conclusions of the papers [16], [17], [25], [26], introduction of the surface tension on the boundary of the semiplane leads to elimination of the power singularities of the stresses and the derivatives of the displacements. Only weaker logarithmic singularities may still be present.

Observe that the conditions (4.2) provide two out of four necessary conditions to find the constants $C_{j}$ in (3.22). The other two conditions can be obtained by fixing the total force acting on the stamp and its moment about the origin:

$$
\int_{-l}^{l} \sigma_{22}(t, 0) d t=Y, \quad \int_{-l}^{l} t \sigma_{22}(t, 0) d t=M=0 .
$$

5. Solution for an adhesive contact problem. Consider a rigid stamp indentation into an elastic semiplane similar to the previous sections. Assume that the friction between the semiplane and the stamp is so large that the stamp and the semiplane are in the adhesive contact with each other. The boundary condition (2.3) is replaced in this case by the following adhesive condition in the contact zone:

$$
u_{1,1}(t, 0)=g_{3}(t), \quad u_{2,1}(t, 0)=g_{2}(t), \quad t \in L_{0},
$$

where the functions $g_{2}(t), g_{3}(t)$ describe the profile of the rigid stamp. The boundary conditions (2.4) outside of the contact zone stay the same. Using a procedure similar to that for frictionless contact, obtain that the conditions (3.10) are replaced with

$$
\operatorname{Im} q_{0}(t)=-\frac{2 \mu}{\kappa+1} g_{3}(t), \quad \operatorname{Re} g_{0}^{\prime}(t)=\frac{2 \mu}{\kappa+1} g_{2}(t), \quad t \in L_{0},
$$

while the conditions (3.11) stay the same.

The integral equations (3.12), (3.13) remain the same, and (3.14) needs to be replaced with

$$
\begin{aligned}
& -\mathcal{F}\left(\operatorname{Re} g_{1}^{\prime}(t)\right)-i \operatorname{sign} s \frac{\gamma_{2}(\kappa+1)}{4 \mu} \mathcal{F}\left(\operatorname{Re} g_{1}^{\prime \prime}(t)\right)+\frac{\gamma_{1}(\kappa-1)}{4 \mu} \mathcal{F}\left(\operatorname{Re} g_{1}^{\prime \prime \prime}(t)\right) \\
& =\frac{2 \mu}{\kappa+1} \mathcal{F}\left(g_{2}(t)\right)-i \operatorname{sign} s \mathcal{F}\left(\operatorname{Re} q_{0}(t)\right)-\frac{\kappa-1}{\kappa+1} \mathcal{F}\left(\operatorname{Im} q_{0}(t)\right) .
\end{aligned}
$$

Copyright $\odot$ by SIAM. Unauthorized reproduction of this article is prohibited. 
Following the solution procedure in the previous sections, the problem can be reduced to two weakly singular integral equations on the finite segment $L_{0}$ with respect to the unknown functions $\operatorname{Re} q_{0}(t)$ and $\operatorname{Im} q_{0}(t)$ :

$$
\begin{gathered}
\frac{2 \kappa}{\kappa+1} \operatorname{Im} q_{0}(t)=-\frac{\gamma_{2} \kappa(\kappa+1)}{2 \pi \mu(\kappa-1)} \int_{L} \frac{\operatorname{Re} g_{1}^{\prime \prime}(\tau) d \tau}{\tau-t} \\
-\frac{(\kappa+1)^{2}}{2 \pi^{2}(\kappa-1)} \int_{L} \log \left|\frac{(l-\tau)(l+t)}{(l+\tau)(l-t)}\right| \frac{\operatorname{Re} g_{1}^{\prime}(\tau) d \tau}{\tau-t}-\frac{\mu(\kappa-1)}{\kappa+1} g_{2}(t) \\
-\frac{\mu}{\pi} \int_{L_{0}} \frac{g_{3}(\tau) d \tau}{\tau-t}-\frac{\mu(\kappa+1)}{\pi^{2}(\kappa-1)} \int_{L_{0}} \log \left|\frac{(l-\tau)(l+t)}{(l+\tau)(l-t)}\right| \frac{g_{2}(\tau) d \tau}{\tau-t}, \quad t \in L_{0},
\end{gathered}
$$

$$
\frac{2 \kappa}{\kappa+1} \operatorname{Re} q_{0}(t)=\frac{\kappa+1}{2 \pi} \int_{L} \frac{\operatorname{Re} g_{1}^{\prime}(\tau) d \tau}{\tau-t}-\frac{\mu(\kappa-1)}{\kappa+1} g_{3}(t)+\frac{\mu}{\pi} \int_{L_{0}} \frac{g_{2}(\tau) d \tau}{\tau-t}, \quad t \in L_{0},
$$

where

$$
\begin{gathered}
\operatorname{Re} g_{1}^{\prime}(t)=-\frac{\mu}{\gamma_{1}(\kappa-1) \pi^{2}} \int_{L_{0}} \operatorname{Re} q_{0}(\tau) K_{2}(t-\tau) d \tau \\
-\frac{\mu}{\gamma_{1}(\kappa+1) \pi^{2}} \int_{L_{0}} \operatorname{Im} q_{0}(\tau) K_{1}(t-\tau) d \tau-\frac{2 \mu^{2}}{\gamma_{1}\left(\kappa^{2}-1\right) \pi^{2}} \int_{L_{0}} g_{2}(\tau) K_{1}(t-\tau) d \tau \\
+\frac{1}{2 \pi} C_{1}\left(K_{3}(t-l)-\frac{\gamma_{2}(\kappa+1)}{2 \gamma_{1}(\kappa-1) \pi} K_{2}(t-l)\right)-\frac{1}{2 \pi} C_{2}\left(K_{3}(t+l)-\frac{\gamma_{2}(\kappa+1)}{2 \gamma_{1}(\kappa-1) \pi} K_{2}(t+l)\right) \\
+\frac{1}{4 \pi^{2}}\left(-C_{3} K_{1}(t-l)+C_{4} K_{1}(t+l)\right), \quad t \in L_{1}, \\
\quad \operatorname{Re} g_{1}^{\prime \prime}(t)=-\frac{2 \mu}{\gamma_{1}(\kappa-1) \pi} \int_{L_{0}} \operatorname{Re} q_{0}(\tau) K_{4}(t-\tau) d \tau \\
+\frac{2 \mu}{\gamma_{1}(\kappa+1) \pi} \int_{L_{0}} \operatorname{Im} q_{0}(\tau) K_{3}(t-\tau) d \tau+\frac{4 \mu^{2}}{\gamma_{1}\left(\kappa^{2}-1\right) \pi} \int_{L_{0}} g_{2}(\tau) K_{3}(t-\tau) d \tau \\
+\frac{\mu}{\gamma_{1}(\kappa-1) \pi^{2}}\left(-C_{1} K_{1}(t-l)+C_{2} K_{1}(t+l)\right)-\frac{1}{2 \pi}\left(-C_{3} K_{3}(t-l)+C_{4} K_{3}(t+l)\right), \quad t \in L_{1},
\end{gathered}
$$

and the kernels $K_{j}(t)$ and the constants $C_{j}$ are defined in the same way as in the case of the frictionless contact.

Assume that the given functions $g_{2}(t), g_{3}(t)$ belong to the space $L^{2}(-l, l)$ and are Hölder continuous on the segment $L_{0}$ up to the end-points. Then (5.3) is a system of two weakly singular equations with $L^{2}([-l, l] \times[-l, l])$ kernels and $L^{2}(-l, l)$ right-hand sides. Hence, using the theory of Fredholm integral equations [9], we can conclude that the solutions $\operatorname{Re} q_{0}(t), \operatorname{Im} q_{0}(t)$ exist and are unique for almost all values of parameters, belong to the space $L^{2}(-l, l)$, and are Hölder continuous on $L_{0}$, with the possible exception of the end-points $t= \pm l$.

Let us investigate the singularities of the obtained solution. Observe that the functions $\operatorname{Re} g_{1}^{\prime}(t)$ and $\operatorname{Re} g_{1}^{\prime \prime}(t)$ (and hence, $\operatorname{Re} q_{1}(t)$ ), as in the case of the frictionless contact, remain bounded at the end-points $t= \pm l$. Then it can be seen from (5.4) that the function $\operatorname{Im} q_{0}(t)$ can have at most a logarithm-squared type of singularity at the end-points $t= \pm l$, while from (5.5) it follows that the function $\operatorname{Re} q_{0}(t)$ can have at most a logarithmic type of singularity. Then from (4.1) we obtain that the function $\operatorname{Im} q_{1}(t)$ has at most a logarithm-squared type of singularity. Finally, from 
(3.12) it can be seen that the function $\operatorname{Im} g_{1}^{\prime}(t)$ has at most a logarithmic singularity as well. This leads to the following properties of the stresses and the derivatives of the displacements:

$$
\begin{gathered}
\sigma_{12}(t, 0)=O\left(\log ^{2}|t \pm l|\right), \quad t \rightarrow \pm l, \\
\sigma_{22}(t, 0)=O(\log |t \pm l|), \quad t \rightarrow \pm l \mp 0, \quad \sigma_{22}(t, 0)=O(1), \quad t \rightarrow \pm l \pm 0 \\
u_{1,1}(t, 0)=O(1), \quad t \rightarrow \pm l \mp 0, \quad u_{1,1}(t, 0)=O(\log |t \pm l|), \quad t \rightarrow \pm l \pm 0 \\
u_{2,1}(t, 0)=O(1), \quad t \rightarrow \pm l,
\end{gathered}
$$

where either all top signs or all bottom signs need to be taken. Observe that just as in the case of frictionless contact, the stresses and the derivatives of the displacements can have at most logarithm-squared singularities.

The conditions (4.2) do not apply to the case of the adhesive contact. Considering (3.13) and remembering that both of the functions $\operatorname{Re} g_{0}^{\prime}(t)$ and $\operatorname{Re} g_{1}^{\prime}(t)$ are bounded at the points $t= \pm l$, we obtain that the following conditions need to be satisfied:

$$
\begin{gathered}
\lim _{t \rightarrow l+0} \frac{\operatorname{Im} g_{1}^{\prime}(t)}{\log |t-l|}=\lim _{t \rightarrow l-0} \frac{\operatorname{Re} q_{0}(t)}{\log |t-l|}, \\
\lim _{t \rightarrow-l-0} \frac{\operatorname{Im} g_{1}^{\prime}(t)}{\log |t+l|}=\lim _{t \rightarrow-l+0} \frac{\operatorname{Re} q_{0}(t)}{\log |t+l|} .
\end{gathered}
$$

The conditions (4.3) remain the same for the adhesive contact case as well.

6. Numerical scheme for the solution of the systems of singular integrodifferential equations. To find a numerical solution to the systems of singular integro-differential equations (3.12), (3.13) with additional conditions (3.10), (3.11) or $(5.2),(3.11)$, approximate the unknown functions $q(t), g^{\prime}(t)$ by Taylor polynomials separately on the lines $L_{0}$ and $L_{1}$. This method has been previously employed in [25], [26] and proven effective in these studies in comparison with the spline collocation method and with the results obtained by approximating the unknown functions by Fourier series.

The details of the numerical scheme of the solution will be presented in the example of the system (3.12), (3.13) with additional relations (3.10), (3.11). First, approximate the unknown functions $\operatorname{Im} g_{0}^{\prime}(t), \operatorname{Re} q_{0}(t), \operatorname{Re} g_{1}^{\prime}(t)$, and $\operatorname{Im} g_{1}^{\prime}(t)$ and the given function $g_{2}(x)$ by truncated Taylor and Laurent series with a given number of terms:

$$
\begin{gathered}
\operatorname{Im} g_{0}^{\prime}(t)=\sum_{k=0}^{N-1} a_{k}^{1} t^{k}, \quad \operatorname{Re} q_{0}(t)=\sum_{k=0}^{N-1} a_{k}^{2} t^{k}, \quad t \in L_{0}, \\
\operatorname{Im} g_{1}^{\prime}(t)=\sum_{k=1}^{N} a_{k}^{3} t^{-k}, \quad \operatorname{Re} g_{1}^{\prime}(t)=\sum_{k=1}^{N+4} a_{k}^{4} t^{-k}, \quad t \in L_{1}, \\
g_{2}(t)=\sum_{k=0}^{N-1} b_{k} t^{k}, \quad t \in L_{0},
\end{gathered}
$$

where $a_{k}^{1}, a_{k}^{2}, a_{k}^{3}, a_{k}^{4}$ are unknown coefficients and $b_{k}$ are given coefficients. 
Observe that since the functions $\operatorname{Re} g_{1}^{\prime}(t), \operatorname{Im} g_{1}^{\prime}(t)$ decay to zero at infinity, it is possible to assume that the free terms in Taylor series expansion (6.2) of these functions are equal to zero. The additional four terms in the expansion (6.2) of the function $\operatorname{Re} g^{\prime}(t)$ correspond to the constants $C_{j}, j=1,2,3,4$. Compute the singular and the regular integrals of the power series terms explicitly:

$$
\begin{array}{cc}
\int_{|r|<l} \frac{r^{k} d r}{r-t}=-\sum_{j=0, j \neq k}^{\infty} \frac{1-(-1)^{j-k}}{j-k} t^{j} l^{k-j}, & |t|<l, \\
\int_{|r|>l} \frac{r^{-k} d r}{r-t}=\sum_{j=0}^{\infty} \frac{1-(-1)^{j+k}}{j+k} t^{j} l^{-j-k}, & |t|<l, \\
\int_{|r|<l} \frac{r^{k} d r}{r-t}=-\sum_{j=1}^{\infty} \frac{1-(-1)^{j+k}}{j+k} t^{-j} l^{j+k}, \quad|t|>l, \\
\int_{|r|>l} \frac{r^{-k} d r}{r-t}=\sum_{j=1, j \neq k}^{\infty} \frac{1-(-1)^{j-k}}{j-k} t^{-j} l^{j-k}, \quad|t|>l .
\end{array}
$$

Substituting the representations (6.1)-(6.3) into the system of the singular integrodifferential equations (3.12), (3.13) and using the formulas (6.4) and the additional equations (3.10), (3.11), obtain the following system of linear algebraic equations for the unknown coefficients $a_{k}^{j}$ in the representations (6.1), (6.2):

$$
\begin{aligned}
& \frac{\kappa+1}{2} a_{k}^{1} l^{k}+\frac{\kappa-1}{2 \pi} \sum_{j=1}^{N+4} a_{j}^{4} l^{-j} \frac{1-(-1)^{j+k}}{j+k}+\frac{\kappa \gamma_{1}}{2 \mu \pi} \sum_{j=1}^{N+4} j(j+1) a_{j}^{4} l^{-j-2} \frac{1-(-1)^{j+k}}{j+k+2} \\
& =\frac{\mu(\kappa-1)}{\pi(\kappa+1)} \sum_{j=0, j \neq k}^{N-1} b_{j} l^{j} \frac{1-(-1)^{k-j}}{k-j}+\frac{\gamma_{0} \kappa}{\pi(\kappa+1)} \sum_{j=2, j \neq k+2}^{N-1} b_{j} l^{j-2} j(j-1) \frac{1-(-1)^{k-j}}{k-j+2}, \\
& k=0, \ldots, N-1, \\
& -\frac{\kappa-1}{2 \pi} \sum_{j=0, j \neq k}^{N-1} a_{j}^{1} l^{j} \frac{1-(-1)^{k-j}}{k-j}+\frac{\kappa-1}{2 \pi} \sum_{j=1}^{N} a_{j}^{3} l^{-j} \frac{1-(-1)^{j+k}}{j+k} \\
& +\frac{2 \kappa}{\pi(\kappa+1)} \sum_{j=0, j \neq k}^{N-1} a_{j}^{2} l^{j} \frac{1-(-1)^{k-j}}{k-j}-\frac{\kappa \gamma_{2}}{2 \mu \pi} \sum_{j=1}^{N+4} a_{j}^{4} l^{-j-1} j \frac{1+(-1)^{j+k}}{j+k} \\
& =\mu b_{k} l^{k}, \quad k=0, \ldots, N-1, \\
& \frac{\kappa+1}{2} a_{k}^{3} l^{-k}+\frac{\kappa-1}{2 \pi} \sum_{j=1, j \neq k}^{N+4} a_{j}^{4} l^{-j} \frac{1-(-1)^{k-j}}{k-j}+\frac{\kappa \gamma_{1}}{2 \mu \pi} \sum_{j=1, j \neq k-2}^{N+4} a_{j}^{4} l^{-j-2} j(j+1) \frac{1-(-1)^{k-j}}{k-j-2} \\
& =\frac{\mu(\kappa-1)}{\pi(\kappa+1)} \sum_{j=0}^{N-1} b_{j} l^{j} \frac{1-(-1)^{j+k}}{j+k}+\frac{\gamma_{0} \kappa}{\pi(\kappa+1)} \sum_{j=2}^{N-1} b_{j} l^{j-2} j(j-1) \frac{1-(-1)^{j+k}}{j+k-2}, k=1, \ldots, N \text {, } \\
& -\frac{\kappa+1}{2} a_{k}^{4} l^{-k}-\frac{\kappa-1}{2 \pi} \sum_{j=0}^{N-1} a_{j}^{1} l^{j} \frac{1-(-1)^{j+k}}{j+k}+\frac{\kappa-1}{2 \pi} \sum_{j=1, j \neq k}^{N} a_{j}^{3} l^{-j} \frac{1-(-1)^{k-j}}{k-j}
\end{aligned}
$$

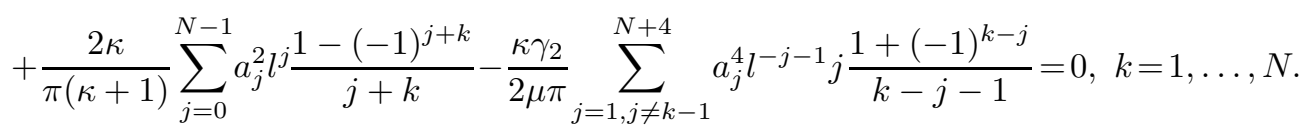

Copyright $@$ by SIAM. Unauthorized reproduction of this article is prohibited. 
To obtain the remaining four equations, substitute the representations (6.1)-(6.3) into the additional conditions (4.2), (4.3):

$$
\begin{gathered}
\sum_{k=0}^{N-1} a_{k}^{2}( \pm l)^{k}=\frac{\gamma_{2}(\kappa+1)}{4 \mu} \sum_{k=1}^{N+4} k a_{k}^{4}( \pm l)^{-k-1}, \\
\sum_{k=0}^{N-1} a_{k}^{2} \frac{l^{k+1}}{k+1}\left(1+(-1)^{k}\right)=\frac{Y}{2}, \quad \sum_{k=0}^{N-1} a_{k}^{2} \frac{l^{k+2}}{k+2}\left(1-(-1)^{k}\right)=0 .
\end{gathered}
$$

Consider the case of adhesive contact. In this case, the unknowns (6.1) need to be replaced with

$$
\operatorname{Im} q_{0}(t)=\sum_{k=0}^{N-1} a_{k}^{1} t^{k}, \quad \operatorname{Re} q_{0}(t)=\sum_{k=0}^{N-1} a_{k}^{2} t^{k}, \quad t \in L_{0},
$$

and the relations (6.3) become

$$
g_{2}(t)=\sum_{k=0}^{N-1} b_{k}^{2} t^{k}, \quad g_{3}(t)=\sum_{k=0}^{N-1} b_{k}^{3} t^{k}, \quad t \in L_{0},
$$

where again $a_{k}^{1}, a_{k}^{2}, a_{k}^{3}, a_{k}^{4}$ are unknown coefficients and $b_{k}^{2}, b_{k}^{3}$ are given coefficients.

Substituting the representations (6.2), (6.8) into the conditions (3.12), (3.13), (3.10), and (5.2), obtain the following system of linear algebraic equations:

$$
\begin{gathered}
-\frac{2 \kappa}{\pi(\kappa+1)} \sum_{j=0, j \neq k}^{N-1} a_{j}^{1} l^{j} \frac{1-(-1)^{k-j}}{k-j}+\frac{\kappa-1}{2 \pi} \sum_{j=1}^{N+4} a_{j}^{4} l^{-j} \frac{1-(-1)^{j+k}}{j+k} \\
+\frac{\gamma_{1} \kappa}{2 \mu \pi} \sum_{j=1}^{N+4} j(j+1) a_{j}^{4} l^{-j-2} \frac{1-(-1)^{j+k}}{j+k+2}=\mu b_{k}^{3} l^{k} \\
+\frac{\mu(\kappa-1)}{\pi(\kappa+1)} \sum_{j=0, j \neq k}^{N-1} b_{j}^{2} l^{j} \frac{1-(-1)^{k-j}}{k-j}, \quad k=0, \ldots, N-1, \\
\frac{2 \kappa}{\pi(\kappa+1)} \sum_{j=0, j \neq k}^{N-1} a_{j}^{2} l^{j} \frac{1-(-1)^{k-j}}{k-j}+\frac{\kappa-1}{2 \pi} \sum_{j=1}^{N} a_{j}^{3} l^{-j} \frac{1-(-1)^{j+k}}{j+k} \\
-\frac{\kappa \gamma_{2}}{2 \mu \pi} \sum_{j=1}^{N+4} a_{j}^{4} l^{-j-1} j \frac{1+(-1)^{j+k}}{j+k+1}=\mu b_{k}^{2} l^{k} \\
-\frac{\mu(\kappa-1)}{\pi(\kappa+1)} \sum_{j=0, j \neq k}^{N-1} b_{j}^{3} l^{j} \frac{1-(-1)^{k-j}}{k-j}, \quad k=0, \ldots, N-1, \\
\frac{\kappa+1}{2} a_{k}^{3} l^{-k+\frac{\kappa-1}{2 \pi}} \sum_{j=1, j \neq k}^{N+4} a_{j}^{4} l^{-j} \frac{1-(-1)^{k-j}}{k-j}+\frac{\kappa \gamma_{1}}{2 \pi \mu} \sum_{j=1, j \neq k-2}^{N+4} a_{j}^{4} l^{-j-2} j(j+1) \frac{1-(-1)^{k-j}}{k-j}
\end{gathered}
$$

Copyright $\odot$ by SIAM. Unauthorized reproduction of this article is prohibited. 


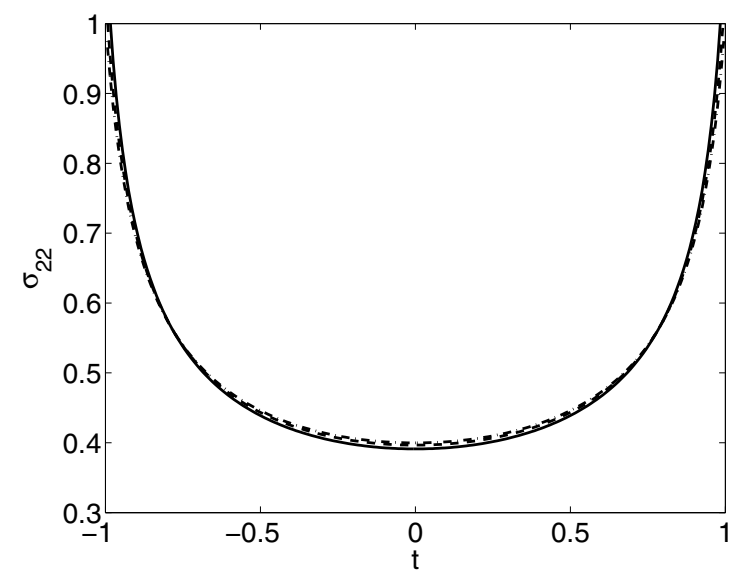

FIG. 2. The normal stress $\sigma_{22}$ on the boundary of the stamp for different numbers $N$ of terms in the series: $N=80, N=100$, and $N=120$.

$$
\begin{aligned}
& -\frac{2 \kappa}{\pi(\kappa+1)} \sum_{j=0}^{N-1} a_{j}^{1} l^{j} \frac{1-(-1)^{k+j}}{k+j}=\frac{\mu(\kappa-1)}{\pi(\kappa+1)} \sum_{j=0}^{N-1} b_{j}^{2} l^{-j} \frac{1-(-1)^{k-j}}{k-j}, \quad k=1, \ldots, N, \\
& \frac{2 \kappa}{\pi(\kappa+1)} \sum_{j=0}^{N-1} a_{j}^{2} l^{j} \frac{1-(-1)^{j+k}}{j+k}-\frac{\kappa+1}{2} a_{k}^{4} l^{-k}+\frac{\kappa-1}{2 \pi} \sum_{j=1, j \neq k}^{N} a_{j}^{3} l^{-j} \frac{1-(-1)^{k-j}}{k-j} \\
& -\frac{\kappa \gamma_{2}}{2 \mu \pi} \sum_{j=1, j \neq k}^{N+4} a_{j}^{4} l^{-j-1} j \frac{1+(-1)^{k-j}}{k-j-1}=-\frac{\mu(\kappa-1)}{\pi(\kappa+1)} \sum_{j=0}^{N-1} b_{j}^{3} l^{j} \frac{1-(-1)^{j+k}}{j+k}, \quad k=1, \ldots, N .
\end{aligned}
$$

The additional conditions (4.2) should be replaced in this case with the conditions (5.6). The meaning of the conditions (5.6) is that the functions $\operatorname{Im} g_{1}^{\prime}(t)$ and $\operatorname{Re} q_{0}(t)$ grow with the same (logarithmic) rate as $t \rightarrow \pm l$. Hence, we can replace the conditions (5.6) with the approximate conditions

$$
\sum_{k=1}^{N} a_{k}^{3}( \pm l)^{-k}=\sum_{k=0}^{N-1} a_{k}^{2}( \pm l)^{k} .
$$

Equations (6.7) constitute the last two equations of the system.

Solving the systems of linear algebraic equations (6.5)-(6.7) and (6.7), (6.10), (6.11) allows us to obtain the approximate solutions of the contact problem for the frictionless and the adhesive cases correspondingly.

7. Numerical results. The computations presented in this section are made for the elastic semiplane with the shear modulus $\mu=70 \mathrm{GPa}$ and the Poisson ratio $\nu=0.33$ (corresponds to aluminum alloy), the surface tension parameters $\gamma_{0}=\gamma_{1}=$ $\gamma_{2}=\gamma$, and the size of the contact area $l=1$.

The convergence results are demonstrated in Figure 2 for the adhesive contact with a different number of terms in the Taylor series approximations (6.2), (6.10), (6.11). The stamp has a straight profile which corresponds to the functions $g_{2}(x)=$ $g_{3}(x)=0$. Other parameters of the problem are $\gamma=0.01$ and $Y=1 \mathrm{MN}$. Here the solid lines correspond to $N=120$, the dashed lines correspond to $N=100$, and the 

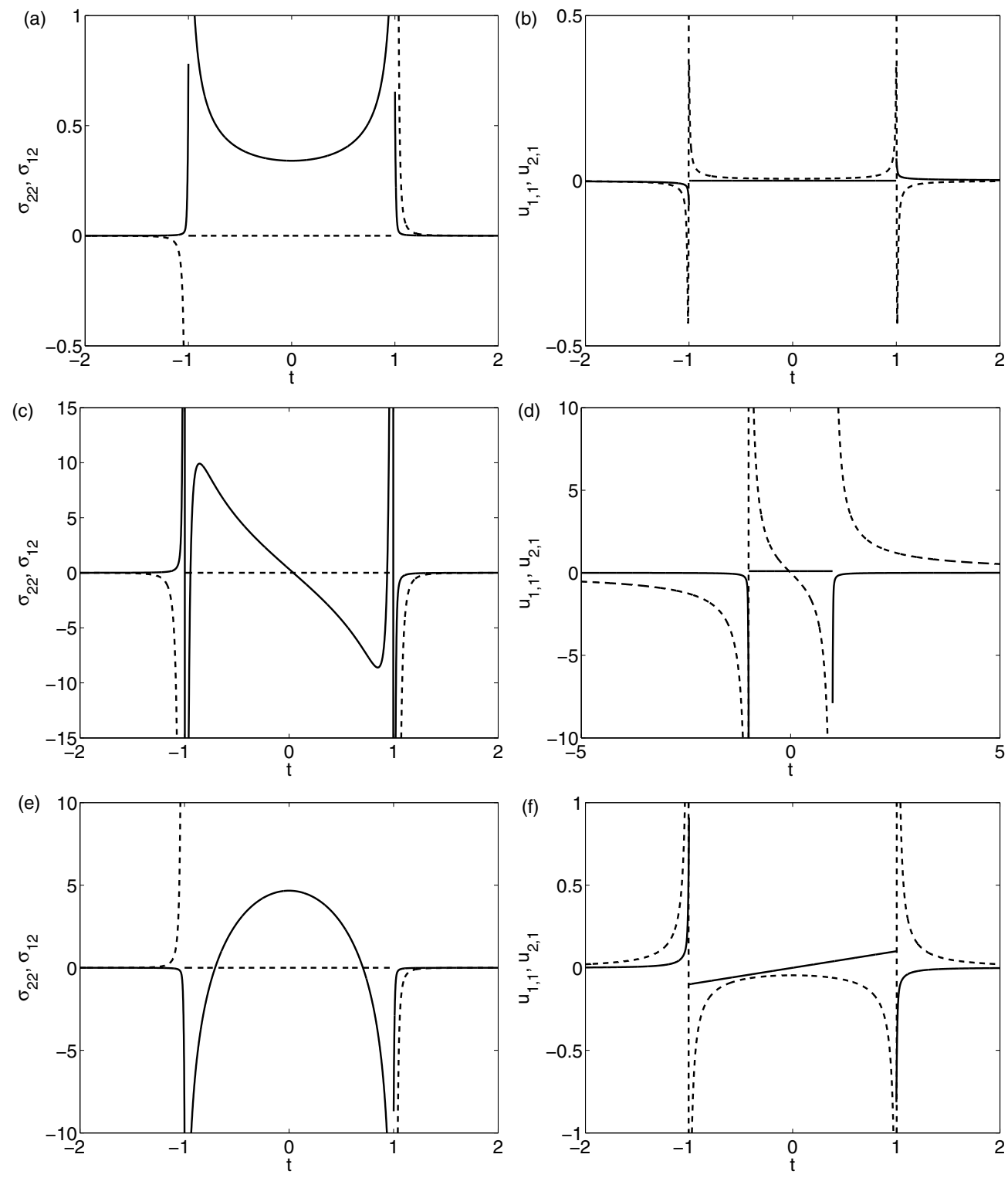

FIG. 3. The stresses and the derivatives of the displacements on the boundary of the semiplane for the straight, the slanted, or the parabolic stamp in the frictionless contact with the semiplane.

dashed-dotted lines correspond to $N=80$. It can be seen that reasonable accuracy is obtained already for $N=80$. To ensure the accuracy of the results for all future computations in this paper, the parameter $N$ is taken to be $N=300$.

The stresses and the derivatives of the displacements for the frictionless contact are plotted in Figure 3 for three different shapes of the stamp profile: straight (Figure $3(\mathrm{a}),(\mathrm{b})$ ), slanted (Figure 3(c),(d)), and parabolic (Figure 3(e),(f)). The graphs are plotted for the semiplane with elastic parameters $\mu=70 \mathrm{GPa}$ and $\nu=0.33$ and the surface tension coefficient $\gamma=0.1$. The straight stamp corresponds to the function 
$g_{2}(t)=0$, the slanted stamp to the function $g_{2}(t)=0.1$, and the parabolic stamp to the function $g_{2}(t)=0.1 t$. The graphs in Figure 3(a),(c),(e) show the normal and the shear stresses on the boundary of the semiplane. The solid lines denote the normal stress $\sigma_{22}$, and the dashed lines denote the stress $\sigma_{12}$. Similarly, the graphs in Figure 3(b),(d),(f) show the derivatives of the displacements, where the solid lines denote the derivative of the displacement $u_{2,1}$, and the dashed lines denote the derivative of the displacement $u_{1,1}$. The vertical force $Y$ acting on the stamp is equal to $Y=1 \mathrm{MN}$ in all cases. Similar graphs for the adhesive case are presented in Figure 4. The notation and the mechanical and the geometric parameters are the same as those in Figure 3. It is assumed that the function $g_{3}(t)=0$ in all of the cases.

The influence of the surface tension parameter $\gamma$ on the normal stresses $\sigma_{22}$ and the derivatives of the displacements $u_{1,1}$ is presented in Figure 5 for frictionless contact of the straight stamp with the semiplane. The shear stresses $\sigma_{12}$ and the derivatives of the displacements $u_{2,1}$ in the case of the frictionless contact are completely defined by the boundary conditions (3.10). The graphs are plotted for the following values of the surface tension parameter $\gamma: \gamma=0.001$ (dotted line), $\gamma=0.01$ (dasheddotted line), $\gamma=0.1$ (dashed line), and the classical contact problem with no surface tension present $(\gamma=0)$ [10]. It appears that the stresses and the derivatives of the displacements change little with the change of the surface tension parameter $\gamma$, except for the areas near the end-points of the stamp, where the surface tension plays a significant role, as expected. Similar graphs for the stresses $\sigma_{22}$ and $\sigma_{12}$ for the case of adhesive contact of the straight stamp with the semiplane are presented in Figure 6 . The derivatives of the displacements $u_{1,1}$ and $u_{2,1}$ are given in this case by the boundary conditions (5.1). Observe also that the values of the stresses and the derivatives of the displacements in the presence of the surface tension do not approach the values for the classical problem as $\gamma \rightarrow 0$. This phenomenon has been noticed previously for the problem of an interface crack [25].

The influence of the surface tension parameter $\gamma$ on the stresses $\sigma_{22}$ in the case of frictionless contact of the slanted stamp with the semiplane is presented in Figure 7. The boundary of the stamp is given by the function $g_{2}(t)=0.1$. The computations are made for the same values of the surface tension parameter $\gamma$ as in Figure 5.

The shape of the boundary of the semiplane is presented in Figure 8 for the straight $g_{2}(t)=0$ (Figure $8(\mathrm{a})$ ) and the parabolic $g_{2}(t)=0.1 t$ (Figure $8(\mathrm{~b})$ ) stamp in the frictionless (solid lines) or the adhesive (dashed lines) contact with the semiplane for different values of the total force $Y$ acting on the stamp.

Finally, the size effects of the surface tension on the solution of the contact problems are presented in Figure 9, which shows the values of the normalized tensile stress $l \sigma_{22} / Y$ on the boundary of the straight stamp for different values of the half-width of the stamp $l$. The dashed-dotted line corresponds to $l=1$, the dashed line to $l=0.5$, and the dotted line to $l=0.1$. The solid line corresponds to the solution of the classical problem with no surface tension present, which is size-independent. Figure 9(a) corresponds to the frictionless contact and Figure 9(b) to the adhesive contact. Thus, surface tension brings a length scale into the contact problems and can account for the observed size-dependency of nanoindentation experiments. The results are obtained for $\mu=70 \mathrm{GPa}, \nu=0.33$, and $\gamma=0.1$. Surface effects of Gurtin-Murdoch surface elasticity in contact problems have been observed recently in [14], [24]. 

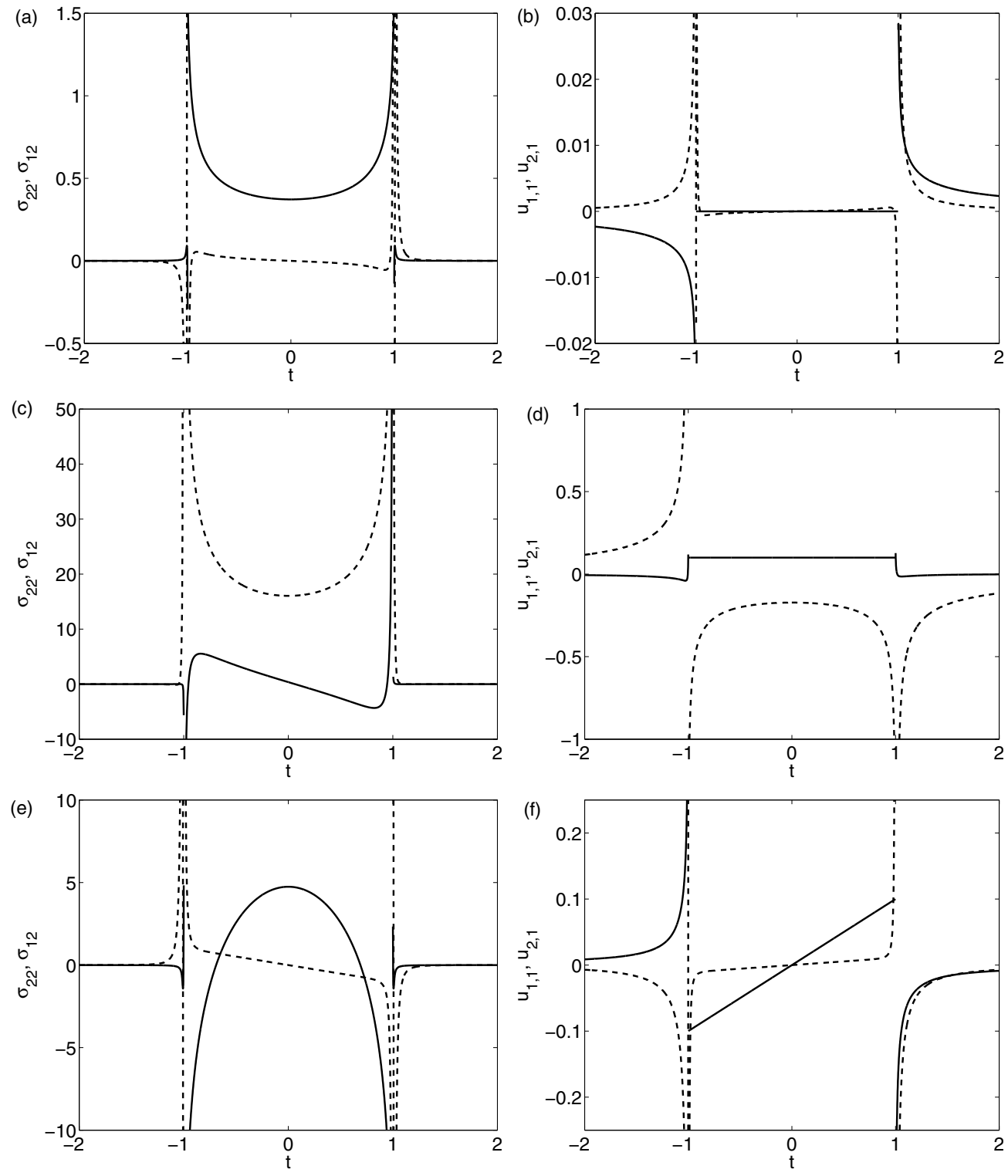

FIG. 4. The stresses and the derivatives of the displacements on the boundary of the semiplane for the straight, the slanted, and the parabolic stamp in the adhesive contact with the semiplane.

8. Conclusions. In this paper, two problems of the frictionless and the adhesive contact of the rigid stamp with the elastic isotropic semiplane have been solved under the assumption that the curvature-dependent surface tension is acting on the boundaries between different materials. The considered surface tension model has been applied previously to the examples of the interface and the noninterface brittle fracture in the studies [16], [17], [25], [26]. 
(a)

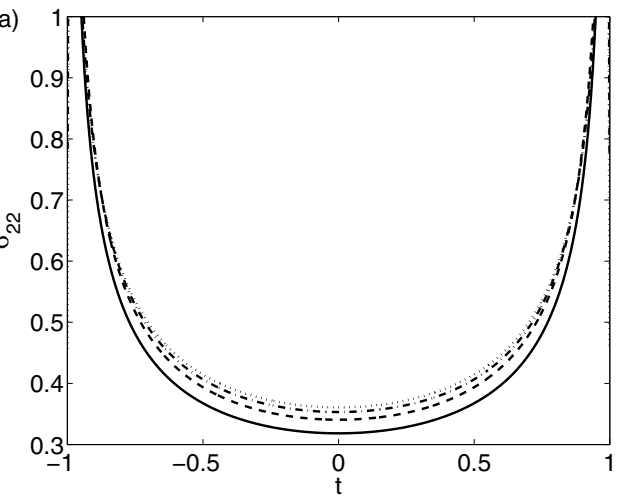

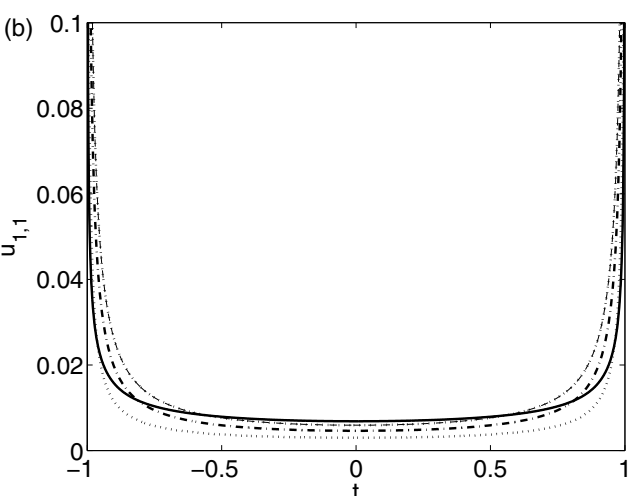

FIG. 5. The stresses $\sigma_{22}$ and the derivatives of the displacements $u_{1,1}$ for the straight stamp in the frictionless contact with the semiplane for different values of the surface tension parameter $\gamma$.

(a)

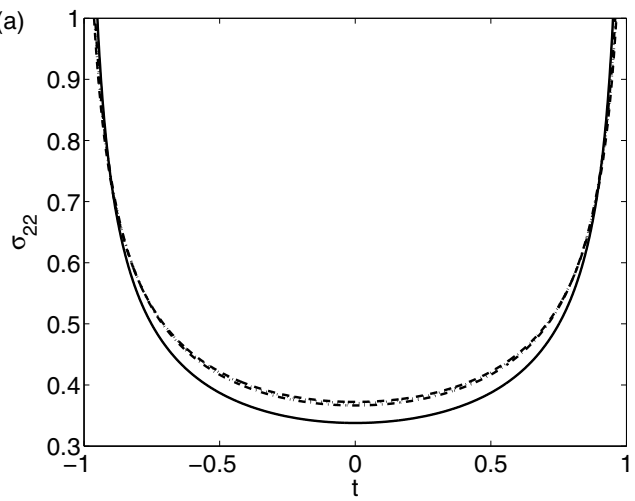

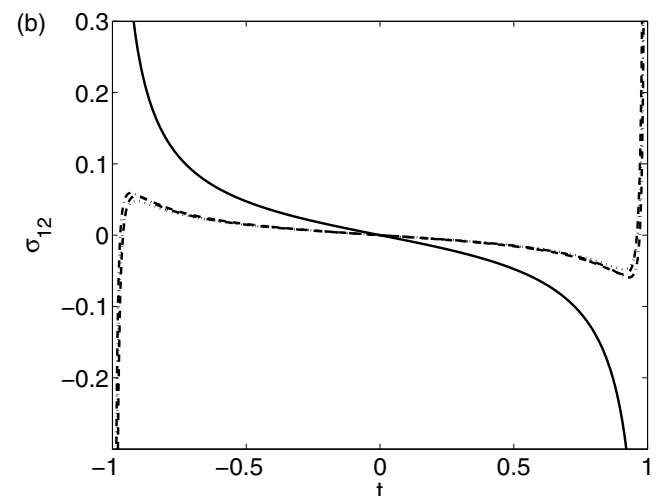

FIG. 6. The stresses $\sigma_{22}, \sigma_{12}$ for the straight stamp in the adhesive contact with the semiplane for different values of the surface tension parameter $\gamma$.

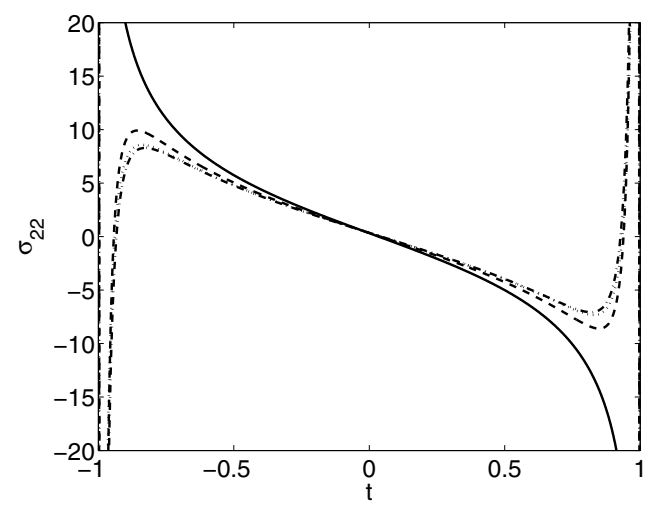

FIG. 7. The stresses $\sigma_{22}$ for the slanted stamp in the frictionless contact with the semiplane for different values of the surface tension parameter $\gamma$.

Copyright $@$ by SIAM. Unauthorized reproduction of this article is prohibited. 
(a)

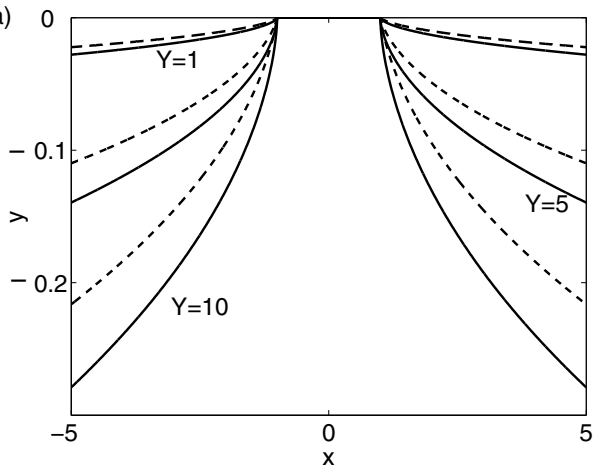

(b)

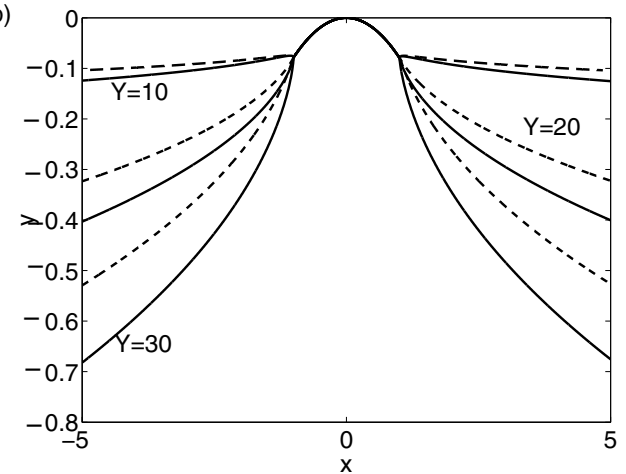

FIG. 8. The shape of the boundary of the semiplane in the frictionless and the adhesive contact with the rigid stamp for different values of the force $Y$ applied to the stamp.
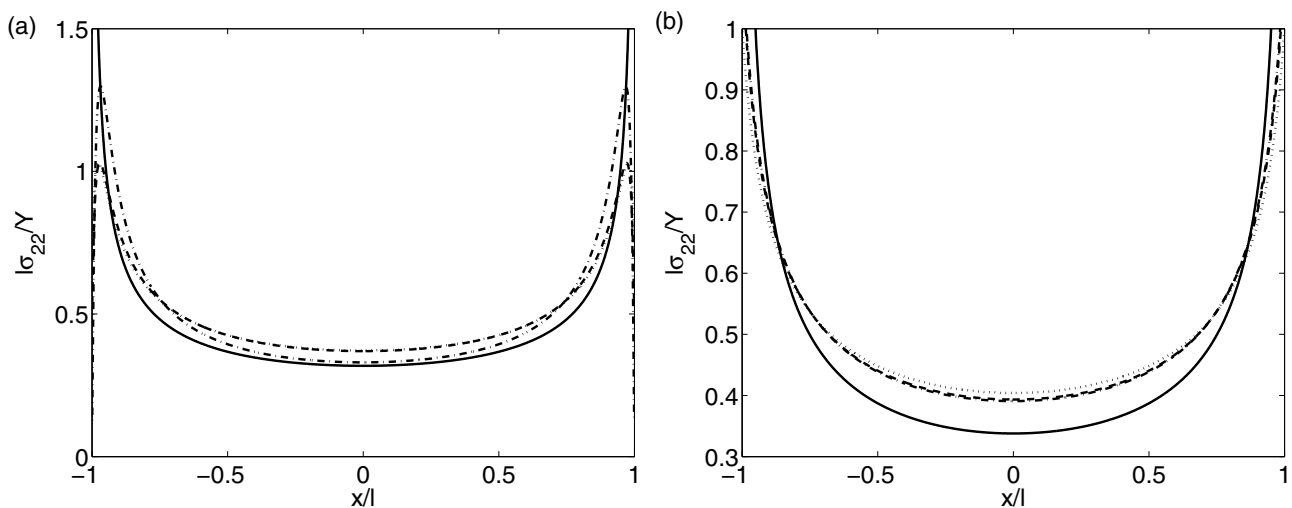

FIG. 9. The normalized stress $l \sigma_{22} / Y$ on the boundary of the straight stamp in the frictionless and the adhesive contact with the semiplane for different values of the half-width of the stamp $l$.

It has been shown here that, similarly to the fracture problems, introduction of the surface tension on the boundary of the semiplane leads to elimination of the integrable power singularities of the order $1 / 2$ at the ends of the contact zone. Weaker logarithmic type singularities may still be present. This fact results in significantly different behavior of the material at the end-points of the contact zone compared to the classical case with no surface tension present on the boundary of the semiplane. In the classical case, provided that the vertical force $Y$ is large enough, the solutions predict that the material will wrap around the "corner" where the stamp surface becomes vertical. In other words, the material of the semiplane separates from the surface of the punch making the angle $\pi / 2$ with the real axis. This nonphysical behavior is not present if the curvature-dependent surface tension is taken into account on the boundary of the semiplane. In fact, the material always separates from the stamp at the end-points of the contact zone with a tangent line parallel to the $x$-axis.

Introduction of the surface mechanics makes the solutions of the contact problems size-dependent. This effect has been observed previously in nanoindentation experiments, and it has been shown that it cannot be accounted for by only using bulk theories. Thus, taking into account the surface tension on the boundaries between 
different materials in the contact problems resolves some of the contradictions of linear elasticity.

An important application of the solutions produced here includes the possibility of obtaining surface tension parameters by comparing the presented theoretical results to the nanoindentation experiments. The values of these parameters can be used after that to solve practically important problems such as, for instance, the problem of fracture propagation.

Finally, development of more general surface tension/surface energy models applicable to various problems of solid mechanics remains an important and challenging practical problem. Satisfactory solution of this problem will necessarily involve a combination of results obtained from physical experiments, ab initio (first principle) computations [18], and mathematical modeling.

Appendix A. Properties of the kernels $K_{j}(t), j=1,2,3,4$. Let us investigate the properties of the kernels $K_{j}(t), j=1,2,3,4$, defined by (3.20). Consider first the function $K_{1}(t)$. Observe that this function is an inverse Fourier mapping of the function $1 /\left\{(|s|-a)^{2}+b^{2}\right\}$ which belongs to both functional spaces $L^{2}(\mathbb{R})$ and $L^{1}(\mathbb{R})$, and hence is continuous and belongs to the space $L^{2}(\mathbb{R})[1]$. Next, consider the difference

$$
\begin{gathered}
\left|K_{1}\left(t_{1}\right)-K_{1}\left(t_{2}\right)\right|=2\left|\int_{0}^{\infty} \frac{\left(\cos 2 \pi s t_{1}-\cos 2 \pi s t_{2}\right) d s}{(s-a)^{2}+b^{2}}\right| \\
\quad \leq 4 \int_{0}^{\infty} \frac{\left|\sin \pi s\left(t_{1}-t_{2}\right)\right|\left|\sin \pi s\left(t_{1}+t_{2}\right)\right| d s}{(s-a)^{2}+b^{2}} .
\end{gathered}
$$

By applying the inequalities $|\sin x| \leq|x|^{\lambda}, 0<\lambda<1$, and $|\sin x| \leq 1$ to the last expression, obtain

$$
\left|K_{1}\left(t_{1}\right)-K_{1}\left(t_{2}\right)\right| \leq 4\left|\pi\left(t_{1}-t_{2}\right)\right|^{\lambda} \int_{0}^{\infty} \frac{s^{\lambda} d s}{(s-a)^{2}+b^{2}}, \quad 0<\lambda<1 .
$$

Observe that the integral $\int_{0}^{\infty} s^{\lambda} d s /\left\{(s-a)^{2}+b^{2}\right\}$ exists for all $0<\lambda<1$ and does not depend on the points $t_{1}, t_{2}$. Hence, from the inequality (A.1), it follows that the function $K_{1}(t)$ is Hölder continuous on the whole real axis with any exponent $\lambda$, $0<\lambda<1$. Observe that it can be shown using a similar procedure that the kernel $K_{2}(t)$ also belongs to $L^{2}(\mathbb{R})$ and is Hölder continuous on the whole real axis with any exponent $\lambda, 0<\lambda<1$. It can be easily shown that Hölder continuity, together with absolute square integrability, implies that $K_{1}(t) \rightarrow 0, K_{2}(t) \rightarrow 0$ as $t \rightarrow \pm \infty$.

Next, notice that the functions $K_{3}(t)$ and $K_{4}(t)$ belong to the space $L^{2}(\mathbb{R})$ as inverse Fourier mappings of the functions from the space $L^{2}(\mathbb{R})$. At the same time, these functions may not necessarily be continuous. For instance, consider the kernel $K_{3}(t)$ :

$$
\begin{gathered}
K_{3}(t)=2 \int_{-a}^{\infty} \frac{(s+a) \sin 2 \pi(s+a) t d s}{s^{2}+b^{2}} \\
=2 \int_{-a}^{0} \frac{s \sin 2 \pi(s+a) t d s}{s^{2}+b^{2}}+2 \cos 2 \pi a t \int_{0}^{\infty} \frac{s \sin 2 \pi s t d s}{s^{2}+b^{2}} \\
+2 \sin 2 \pi a t \int_{0}^{\infty} \frac{s \cos 2 \pi s t d s}{s^{2}+b^{2}}+2 a \int_{0}^{\infty} \frac{\sin 2 \pi s t d s}{(s-a)^{2}+b^{2}} \\
=2 I_{1}(t)+2 I_{2}(t) \cos 2 \pi a t+2 I_{3}(t) \sin 2 \pi a t+2 a I_{4}(t) .
\end{gathered}
$$

Copyright $@$ by SIAM. Unauthorized reproduction of this article is prohibited. 
Observe that the integral $I_{1}(t)$ is a continuously differentiable function on the real line $\mathbb{R}$ with a bounded derivative and hence is a Lipschitz continuous function. The integral $I_{2}(t)$ can be computed explicitly [3],

$$
I_{2}(t)=\int_{0}^{\infty} \frac{s \sin 2 \pi s t d s}{s^{2}+b^{2}}=\frac{\pi}{2} e^{-2 \pi b|t|} \operatorname{sign} t,
$$

and is a continuously differentiable function on the intervals $(-\infty, 0)$ and $(0, \infty)$ with a bounded derivative. Hence, it is Lipschitz continuous on these intervals.

Consider the third integral $I_{3}(t)[3]$,

$$
I_{3}(t)=\int_{0}^{\infty} \frac{s \cos 2 \pi s t d s}{s^{2}+b^{2}}=-\frac{1}{2}\left[e^{-2 \pi b|t|} \operatorname{Ei}(2 \pi b|t|)+e^{2 \pi b|t|} \operatorname{Ei}(-2 \pi b|t|)\right] .
$$

Here $\operatorname{Ei}(x)$ denotes the exponential integral function [3],

$$
\operatorname{Ei}(x)=-\int_{-x}^{\infty} \frac{e^{-t}}{t} d t=\gamma+\log |x|+\sum_{k=1}^{\infty} \frac{x^{k}}{k \cdot k !}, \quad x \neq 0,
$$

where $\gamma$ is the Euler constant, $\gamma=0.57721 \ldots$, and

$$
\operatorname{Ei}^{\prime}(x)=e^{x} / x .
$$

Hence, the integral $I_{3}(t)$ has a logarithmic singularity at $t=0$ and is continuous at all other points. Also, differentiating the right-hand side of the equality (A.3) by $t$, obtain

$$
\frac{d}{d t}\left(\int_{0}^{\infty} \frac{s \cos 2 \pi s t d s}{s^{2}+b^{2}}-2 \log |t|\right)=2 \pi b^{2} \operatorname{sign} t \int_{0}^{\infty} \frac{\sin 2 \pi s t d s}{s^{2}+b^{2}}
$$

where the right-hand side is a continuous bounded function. Thus, the function $I_{3}(t)-2 \log |t|$ is a Lipschitz continuous function.

Finally, using the same procedure as for the function $K_{1}(t)$, obtain that the integral $I_{4}(t)$ is a Hölder continuous function on $\mathbb{R}$ with any exponent $\lambda, 0<\lambda<1$.

Hence, combining everything together, it can be deduced that $K_{3}(t)$ is a Hölder continuous function on the intervals $(-\infty, 0)$ and $(0, \infty)$ with any exponent $\lambda, 0<$ $\lambda<1$. At the point $t=0$ this function has a jump discontinuity.

In a similar way, consider the function $K_{4}(t)$,

$$
\begin{gathered}
K_{4}(t)=2 \int_{-a}^{\infty} \frac{(s+a) \cos 2 \pi(s+a) t d s}{s^{2}+b^{2}} \\
=2 \int_{-a}^{0} \frac{s \cos 2 \pi(s+a) t d s}{s^{2}+b^{2}}-2 \sin 2 \pi a t \int_{0}^{\infty} \frac{s \sin 2 \pi s t d s}{s^{2}+b^{2}} \\
+2 \cos 2 \pi a t \int_{0}^{\infty} \frac{s \cos 2 \pi s t d s}{s^{2}+b^{2}}+2 a \int_{0}^{\infty} \frac{\cos 2 \pi s t d s}{(s-a)^{2}+b^{2}} \\
=2 J_{1}(t)-2 I_{2}(t) \sin 2 \pi a t+2 I_{3}(t) \cos 2 \pi a t+2 a J_{4}(t),
\end{gathered}
$$

where the functions $J_{1}(t)$ and $J_{4}(t)$ have the same properties as the functions $I_{1}(t)$ and $I_{4}(t)$. Hence, it can be concluded that the kernel $K_{4}(t)$ is a Hölder continuous function on the intervals $(-\infty, 0)$ and $(0, \infty)$ with any exponent $\lambda, 0<\lambda<1$. At the point $t=0$ this function has a logarithmic discontinuity. For the same reasons as previously, $K_{3}(t) \rightarrow 0, K_{4}(t) \rightarrow 0$ as $t \rightarrow \infty$. 


\section{REFERENCES}

[1] H. Dym and H. McKean, Fourier Series and Integrals, Academic Press, New York, 1972.

[2] F. Gakhov, Boundary Value Problems, Dover Publications, New York, 1990.

[3] I. Gradshteyn and I. Ryzhik, Table of Integrals, Series and Products, 7th ed., Academic Press, New York, 2007.

[4] M. Gurtin and A. Murdoch, A continuum theory of elastic material surfaces, Arch. Rational Mech. Anal., 57 (1975), pp. 291-323.

[5] M. Gurtin And A. Murdoch, Surface stress in solids, Int. J. Solids Struct., 14 (1978), pp. 431440.

[6] L. He And C. Lim, Surface Green function for a soft elastic half-space: Influence of surface stress, Int. J. Solids Struct., 43 (2011), pp. 217-235.

[7] M. Horstemeyer and M. Baskes, Atomistic finite deformation simulations: A discussion on length scale effects in relation to mechanical stresses, Trans. ASME, 121 (1999), pp. 114119.

[8] F. King, Hilbert Transforms. Vol. 1, Encyclopedia Math. Appl. 24, Cambridge University Press, Cambridge, UK, 2009.

[9] M. Krasnov, Integral Equations: Introduction into the Theory, Nauka, Moscow, 1975 (in Russian).

[10] N. Muskhelishvili, Some Basic Problems of the Mathematical Theory of Elasticity: Fundamental Equations, Plane Theory of Elasticity, Torsion and Bending, translated from the Russian by J. R. M. Radok, P. Noordhoff, Ltd., Groningen, 1963.

[11] W. Nix AND H. GaO, Indentation size effects in crystalline materials: A law for strain gradient plasticity, J. Mech. Phys. Solids, 46 (1998), pp. 411-425.

[12] E.-S. Оh, J. Walton, AND J. Slattery, A theory of fracture based upon an extension of continuum mechanics to nanoscale, J. Appl. Mech., 73 (2006), pp. 792-780.

[13] W. Oliver AND G. PharR, An improved technique for determining hardness and elastic modulus using load and displacement sensing indentation experiments, J. Mater. Res., 7 (1992), pp. 1564-1583.

[14] Y. Pinyochotiwong, J. Rungamornrat, and T. Senjuntichai, Analysis of rigid frictionless indentation on half-space with surface elasticity, Procedia Engrg., 14 (2011), pp. 24032410 .

[15] M. Savruk, Two-Dimensional Problems of Elasticity for Cracked Solids, Naukova Dumka, Kiev, 1981 (in Russian).

[16] T. Sendova And J. Walton, The effect of surface tension in modeling interfacial fracture, in Application of Mathematics in Technical and Natural Sciences, AIP Conf. Proc. 1301, Amer. Inst. Phys., Melville, NY, 2010, pp. 291-300.

[17] T. Sendova And J. R. Walton, A new approach to the modeling and analysis of fracture through extension of continuum mechanics to the nanoscale, Math. Mech. Solids, 15 (2010), pp. 368-413.

[18] H. Skriver and N. Rosengaard, Surface energy and work function of elemental metals, Phys. Rev., 46 (1992), pp. 7157-7168.

[19] J. Slattery, E.-S. OH, AND K.-B. Fu, Extension of continuum mechanics to the nanoscale, Chem. Engrg. Sci., 59 (2004), pp. 4621-4635.

[20] D. Steigmann and R. Ogden, Plane deformations of elastic solids with intrinsic boundary elasticity, Proc. Roy. Soc. London Ser. A, 453 (1997), pp. 853-877.

[21] D. Steigmann and R. Ogden, Elastic surface-substrate interactions, R. Soc. Lond. Proc. Ser. A Math. Phys. Eng. Sci., 455 (1999), pp. 437-474.

[22] J. Walton, Plain-strain fracture with curvature-dependent surface tension: Mixed mode loading, J. Elasticity, 114 (2014), pp. 127-142.

[23] C. Wang And X. Feng, Effects of surface stress on contact problems at nanoscale, J. Appl. Phys., 101 (2007), 013510.

[24] J. R. Y. Pinyochotiwong and T. Senjuntichai, Rigid frictionless indentation on elastic half space with influence of surface stresses, Int. J. Engrg. Sci., 71 (2013), pp. 15-35.

[25] A. Zemlyanova, The effect of a curvature-dependent surface tension on the singularities at the tips of a straight interface crack, Quart. J. Mech. Appl. Math., 66 (2013), pp. 199-219.

[26] A. Zemlyanova and J. R. Walton, Modeling of a curvilinear planar crack with a curvaturedependent surface tension, SIAM J. Appl. Math., 72 (2012), pp. 1474-1492. doi:10.1137/ 110860100

[27] X. ZhaO AND R. RAJAPAKSe, Analytical solutions for a surface-loaded isotropic elastic layer with surface energy effects, Int. J. Engrg. Sci., 47 (2009), pp. 1433-1444.

[28] S. Zhou AND X.-L. GAO, Solutions of half-space and half-plane contact problems based on surface elasticity, Z. Angew. Math. Phys., 64 (2013), pp. 145-166.

Copyright (c) by SIAM. Unauthorized reproduction of this article is prohibited. 